\title{
ON THE 4D NONLINEAR SCHRÖDINGER EQUATION WITH COMBINED TERMS UNDER THE ENERGY THRESHOLD
}

\author{
CHANGXING MIAO, TENGFEI ZHAO, AND JIQIANG ZHENG
}

\begin{abstract}
In this paper, we consider the longtime dynamics of the solutions to focusing energy-critical Schrödinger equation with a defocusing energysubcritical perturbation term under a ground state energy threshold in four spatial dimension. This extends the results in Miao et al. (Commun Math Phys 318(3):767-808, 2013, The dynamics of the NLS with the combined terms in five and higher dimensions. Some topics in harmonic analysis and applications, advanced lectures in mathematics, ALM34, Higher Education Press, Beijing, pp 265-298, 2015) to four dimension without radial assumption and the proof of scattering is based on the interaction Morawetz estimates developed in Dodson (Global well-posedness and scattering for the focusing, energy-critical nonlinear Schröinger problem in dimension $d=4$ for initial data below a ground state threshold, arXiv:1409.1950), the main ingredients of which requires us to overcome the logarithmic failure in the double Duhamel argument in four dimensions.
\end{abstract}

\section{INTRODUCTION}

In this paper, we consider the nonlinear Schrödinger equation with combined terms in four spatial dimension, i.e.

$$
\left\{\begin{aligned}
i \partial_{t} u+\Delta u & =-|u|^{2} u+|u|^{\frac{4}{3}} u, \quad(t, x) \in \mathbb{R} \times \mathbb{R}^{4} \\
u(0, x) & =\varphi(x) \in H^{1}\left(\mathbb{R}^{4}\right),
\end{aligned}\right.
$$

where $\Delta=\sum_{1 \leq j \leq 4} \partial_{x_{j}}^{2}$ is the Laplace operator.

If $u$ is a solution to (CNLS),

$$
M(u)=\int_{\mathbb{R}^{4}}|u|^{2} d x \text { and } E(u)=\int_{\mathbb{R}^{4}}\left[\frac{1}{2}|\nabla u|^{2}-\frac{1}{4}|u|^{4}+\frac{3}{10}|u|^{\frac{10}{3}}\right] d x
$$

are often said to be the mass and energy of $u$ respectively. Through a standard method, one can check that the mass and energy of a smooth solution to (CNLS) conserve in time. The nonlinear term $|u|^{2} u$ is $\dot{H}^{1}\left(\mathbb{R}^{4}\right)$ critical and $|u|^{4 / 3} u$ is $\dot{H}^{1 / 2}\left(\mathbb{R}^{4}\right)$ critical according to the standard scaling analysis.

In general, the energy critical NLS is given by

$$
\left\{\begin{aligned}
i \partial_{t} u+\Delta u & =\mu|u|^{\frac{4}{d-2}} u, \quad(t, x) \in \mathbb{R} \times \mathbb{R}^{d} \\
u(0, x) & =\varphi(x) \in \dot{H}^{1}\left(\mathbb{R}^{d}\right)
\end{aligned}\right.
$$

2000 Mathematics Subject Classification. Primary: 35L70, Secondary: 35Q55.

Key words and phrases. Nonlinear Schrödinger Equation; Longtime dynamics; Interaction Morawetz esimates Scattering; Threshold Energy. 
where $\mu= \pm 1$ and $d \geq 3$. For the local well-posedness of (NLS) in $\dot{H}^{1}\left(\mathbb{R}^{d}\right)$ or $H^{1}\left(\mathbb{R}^{d}\right)$, we refer to $[6,7,8]$.

If $\mu=1$, we call the Cauchy problem (NLS) is defocusing. There are many results in the literature considering the defocusing cases. In [5], Bourgain first developed a method of reduction of energy and proved that any solution to defocusing (NLS) with radial initial data is scattering in spatial dimension three. Colliander. etc. 11] removed the radial assumption by exploiting a interaction Morawetz estimate, and we refer [21] for another proof, which is based on the long time Strichartz estimates. This result was extended in Ryckman and Visan 27. for dimension four (for another proof, we refer to [33])and Visan [32] for five and higher dimensions by using the Morawetz estimates.

For the focusing case $(\mu=-1)$, Kenig and Merle [18 gave out a decomposition of the region if the energy of the solutions under a ground state threshold for radial solutions and they proved that in the dimensions $d \in\{3,4,5\}$, one of the two regions for global well-posedness and scattering and the other region for finite time blow-up. The corresponding results in the cases of five and higher dimensions were proved by Killip and Visan in 20] without radial assumption by employing the double Duhamel formula trick. Dodson 13 obtained global well-posedness and scattering results in dimension four under a ground state threshold by developing the long time Strichartz estimates. But the analog nonradial case of dimension three remains open up to now.

For scattering results of the defocusing energy-subcritical NLS, we refer to [10, [12, 14, 25, 26, 30] and the reference therein.

There are also a quantity of results for the Cauchy problem (NLS) with a energysubcritical nonlinearity perturbation. In 31, Tao, Visan, and Zhang proved the scattering results in $H^{1}\left(\mathbb{R}^{d}\right)$ of defocusing (NLS) with a defocusing perturbation $|u|^{p} u\left(\frac{4}{d} \leq p<\frac{4}{d-2}\right.$ and $\left.d \geq 3\right)$ or with focusing $|u|^{p} u\left(\frac{4}{d} \leq p<\frac{4}{d-2}\right.$ and $\left.d \geq 3\right)$ and the small mass condition. The latter results was extended in Killip, Oh, Pocovnicu, and Visan [19], where the scattering results were obtained for the solutions to the defocusing (NLS) with a focusing term $|u|^{2} u$ in three spatial dimension if their initial datum belong to a certain region given by rescaling.

Miao, $\mathrm{Xu}$, and Zhao [23] proved scattering and finite time blowup results for the focusing (NLS) perturbed by a $\dot{H}^{1 / 2}$-critical defocusing term in spatial dimension three. More precisely, for every radial initial data $u_{0} \in H^{1}\left(\mathbb{R}^{3}\right)$ below a ground state threshold, the corresponding solution $u$ is globally well-posed and scattering, if its energy functional is nonnegative or blows up at finite time, if its energy functional is negative. And in 24], they extends the result to five and higher spatial dimension without the radial assumption. We refer to [2, 3, 4, for the focusing energy critical NLS with some focusing perturbation terms.

In this article, we consider the longtime dynamics behavior of (CNLS) below the energy threshold. First, we consider the variational derivation of the energy. As in [16], we denote

$$
\varphi_{\alpha, \beta}^{\lambda}(x)=e^{\alpha \lambda} \varphi\left(e^{-\beta \lambda} x\right)
$$

where $(\alpha, \beta, \lambda) \in \mathbb{R}^{3}$ and $\varphi: \mathbb{R}^{4} \rightarrow \mathbb{C}$. We will restrict $(\alpha, \beta)$ in a region,

$$
\Omega=\{(\alpha, \beta): \alpha \geq 0,5 \alpha+6 \beta \geq 0,(\alpha, \beta) \neq(0,0)\} .
$$


And for any functional $F$ of $H^{1}\left(\mathbb{R}^{4}\right)$, we define the variation derivation by

$$
\mathcal{L}_{\alpha, \beta} F(\phi)=\left.\frac{d}{d \lambda}\right|_{\lambda=0} F\left(\phi_{\alpha, \beta}^{\lambda}\right) .
$$

For each $(\alpha, \beta) \in \Omega$, we define

$K_{\alpha, \beta}(\phi)=\mathcal{L}_{\alpha, \beta} E(\phi)=(\alpha+\beta) \int\left[|\nabla \phi(x)|^{2}-|\phi(x)|^{4}\right] d x+\left(\alpha+\frac{6}{5} \beta\right) \int|\phi(x)|^{\frac{10}{3}} d x$,

whenever $\phi$ is a function in $H^{1}\left(\mathbb{R}^{4}\right)$. Consider the minimization problem

$$
m_{\alpha, \beta}=\inf \left\{E(\varphi): \varphi \in H^{1}\left(\mathbb{R}^{4}\right) \backslash\{0\}, \quad K_{\alpha, \beta}(\varphi)=0\right\} .
$$

Since the nonlinear term in (CNLS) is $\dot{H}^{1}$-critical growth with the $\dot{H}^{1}$-subcritical perturbation, we will use the modified energy later

$$
E_{c}(u)=\int_{\mathbb{R}^{4}}\left(\frac{1}{2}|\nabla u|^{2}-\frac{1}{4}|u|^{4}\right) d x .
$$

In this paper we will study the solutions to (CNLS) which start from the following two regions below the minimum $m_{\alpha, \beta}$,

$$
\mathcal{K}_{\alpha, \beta}^{+}=\left\{\varphi \in H^{1}\left(\mathbb{R}^{4}\right): E(\varphi)<m_{\alpha, \beta}, K_{\alpha, \beta}(\varphi) \geq 0\right\}
$$

and

$$
\mathcal{K}_{\alpha, \beta}^{-}=\left\{\varphi \in H^{1}\left(\mathbb{R}^{4}\right): E(\varphi)<m_{\alpha, \beta}, K_{\alpha, \beta}(\varphi)<0\right\},
$$

where $(\alpha, \beta) \in \Omega$.

Before stating the main theorem, we give the definition of scattering which will be used later. A global solution $u$ to (CNLS) with $u(0)=u_{0} \in H^{1}\left(\mathbb{R}^{4}\right)$ is scattering, if there exist $u_{ \pm} \in H^{1}\left(\mathbb{R}^{4}\right)$ such that

$$
\lim _{t \rightarrow \pm \infty}\left\|u(t)-e^{i t \Delta} u_{ \pm}\right\|_{H^{1}\left(\mathbb{R}^{4}\right)}=0 .
$$

Now we state our main theorem, which describes the dynamics of (CNLS) under the threshold $m_{\alpha, \beta}$.

Theorem 1.1. Let $(\alpha, \beta) \in \Omega$. For each $\varphi \in \mathcal{K}_{\alpha, \beta}^{+}$, we have that if $u$ is the solution to (CNLS) with $u(0, x)=\varphi$, then $u$ is global well-posed and scattering in $H^{1}\left(\mathbb{R}^{4}\right)$. On the other hand, if $\varphi$ is a radial function in $\mathcal{K}_{\alpha, \beta}^{-}$, then the solution $u$ with the initial data $\varphi$ will blow up at finite time.

Remark 1.2. By using the same argument in this paper one can also obtain the corresponding results of (CNLS) if $|u|^{4 / 3} u$ is replaced by some more general defocusing $|u|^{p} u(1<p<2)$ terms.

To prove the main theorem, we need the following property of the minimal $m_{\alpha, \beta}$.

Proposition 1.3. For $(\alpha, \beta) \in \Omega$, we have

$$
m_{\alpha, \beta}=E_{c}(W)=\frac{1}{4}\|\nabla W\|_{L^{2}\left(\mathbb{R}^{4}\right)}^{2},
$$

where $W(x)$ is the ground state of the massless equation $-\Delta \varphi=|\varphi|^{2} \varphi$, given by $W(x)=\left(1+\frac{|x|^{2}}{8}\right)^{-1}$.

We remark that this proposition implies that $m_{\alpha, \beta}$ is independent of $(\alpha, \beta)$. We also have the properties of $\mathcal{K}_{\alpha, \beta}^{+}$and $\mathcal{K}_{\alpha, \beta}^{-}$,

Proposition 1.4. The regions $\mathcal{K}_{\alpha, \beta}^{+}$and $\mathcal{K}_{\alpha, \beta}^{-}$do not depend on $(\alpha, \beta)$ if $(\alpha, \beta) \in \Omega$. 
Based on this property, we can denote $\mathcal{K}^{+}=\mathcal{K}_{\alpha, \beta}^{+}$and $\mathcal{K}^{-}=\mathcal{K}_{\alpha, \beta}^{-}$for simplicity. We will also prove the energy trapping property which manifest another important property of the regions $\mathcal{K}^{+}$and $\mathcal{K}^{-}$.

Proposition 1.5. Let $u: I \times \mathbb{R}^{4} \rightarrow \mathbb{C}$ be the solution to (CNLS) with initial data $u(0, x)=u_{0}(x) \in H^{1}\left(\mathbb{R}^{4}\right)$. Then, we have

(1) If $u_{0} \in \mathcal{K}^{-}$, then for each $t \in I, u(t) \in \mathcal{K}^{-}$and

$$
\|\nabla u(t)\|_{L_{x}^{2}}>\|\nabla W\|_{L_{x}^{2}}
$$

(2) If $u_{0} \in \mathcal{K}^{+}$, then for each $t \in I, u(t) \in \mathcal{K}^{+}$and

$$
\|\nabla u(t)\|_{L_{x}^{2}}<\|\nabla W\|_{L_{x}^{2}}
$$

This and the energy conservation law shows that if $u$ is a solution to (CNLS) with the initial data $u(0, x) \in \mathcal{K}^{+}$or $\mathcal{K}^{-}$, the solution flow $\{u(t), t \in I\}$ will remain in the regions $\mathcal{K}^{+}$or $\mathcal{K}^{-}$, where $I$ is the maximal lifespan of $u$. Hence this proposition brings about many conveniences to our proof.

To prove the blowup results, we use the same method as in [18, 23, 24]. Indeed, the estimation of the differentials of the localized virial identity helps us preclude the global existence of the solution which starts from $\mathcal{K}^{-}$.

On the other hand, to prove the scattering results, by local well-posedness theory in Section 2, it suffices to show that the global scattering size of $u \in \mathcal{K}^{+}$is bounded by certain constant. To this end, we turn to a proof of contradiction. More precisely, suppose the energy threshold $E_{*}$ is less than $m$, thus there exists a sequence of solutions $u_{n}$ in $\mathcal{K}^{+}$, with the property that $E\left(u_{n}\right) \rightarrow E_{*}$ and $\left\|u_{n}\right\|_{S T(\mathbb{R})} \rightarrow \infty$ as $n \rightarrow \infty$, where $S T(\mathbb{R})$ is the scattering norm we will define later.

By making use of the linear and nonlinear profile decomposition and the stability lemma given by [3], we can obtain a critical element $u_{c}(t, x)$. We will also prove a crucial compactness property of the critical element dynamics, that is, $\left\{u_{c}(t), 0 \leq\right.$ $t<\infty\}$ is precompact in $\dot{H}^{s}\left(\mathbb{R}^{4}\right)$ after module the translation symmetry, for all $s \in(0,1]$.

Finally, in the last step, extinction of the critical element $u_{c}$, we use the interaction Morawetz estimates to deduce that $u_{c}$ actually vanishes. This is a contradiction to the the local well-poesdness theory(which implies that the solution with small initial data is global well-posed and scattering). It is worthwhile to note that, since we consider the scattering problem in $H^{1}\left(\mathbb{R}^{4}\right)$, the mass of $u_{c}$ conserves with time and remains bounded, which makes this step more easier than the analog step in [13. This is the main reason that why we do not use the longtime Strichartz estimates for the critical element $u_{c}$.

This paper is organized as follows: In the first part of Section 2, we give the notations in this paper and recall some basic harmonic analysis tools and the localwellposed theory of (CNLS). We prove Proposition 1.3 1.5 by introducing the variation method in the second part of Section 2. Section 3 will prove the finite time blowup for solutions in $\mathcal{K}^{-}$. In Section 4, we will construct the linear and nonlinear profile decomposition of the solutions sequence to (CNLS). In Section 5, we will finish the proof of the main theorem. 


\section{BASIC ESTIMATES AND VARIATIONAL METHOD}

2.1. Basic Tools. First we will present some harmonic analysis tools which will be used later. We define the Fourier transform on $\mathbb{R}^{4}$ by

$$
\widehat{f}(\xi):=\frac{1}{4 \pi^{2}} \int_{\mathbb{R}^{4}} e^{-i x \cdot \xi} f(x) d x .
$$

Based on this, for each $s \in \mathbb{R}$, we define the differentiation operator $\phi(\nabla)$ by $\widehat{\varphi(\nabla) f}(\xi)=\varphi(i \xi) \hat{f}(\xi)$. Hence we can define the homogeneous Sobolev norms by $\|u\|_{\dot{H}^{s}\left(\mathbb{R}^{4}\right)}=\left\||\nabla|^{s} u\right\|_{L^{2}\left(\mathbb{R}^{4}\right)}$ and the inhomogeneous Sobolev norms by $\|u\|_{H^{s}\left(\mathbb{R}^{4}\right)}^{2}=$ $\|u\|_{\dot{H}^{s}\left(\mathbb{R}^{4}\right)}^{2}+\|u\|_{L^{2}\left(\mathbb{R}^{4}\right)}^{2}$, for $s \in \mathbb{R}$.

We will also use the following two lemmas, which deal with the fractional derivatives.

Lemma 2.1 (Fractional product rule, [9]). Let $s \in(0,1]$ and $f, g \in \mathcal{S}\left(\mathbb{R}^{4}\right)$, then we have

$$
\left\||\nabla|^{s}(f g)\right\|_{L^{p}\left(\mathbb{R}^{4}\right)} \lesssim\left\||\nabla|^{s} f\right\|_{L^{q_{1}\left(\mathbb{R}^{4}\right)}}\|g\|_{L^{r_{1}\left(\mathbb{R}^{4}\right)}}+\left\|\left|f\left\|_{L^{q_{2}\left(\mathbb{R}^{4}\right)}}\right\| \nabla\right|^{s} g\right\|_{L^{r_{2}\left(\mathbb{R}^{4}\right)}}
$$

where $\frac{1}{p}=\frac{1}{q_{1}}+\frac{1}{r_{1}}, \frac{1}{p}=\frac{1}{q_{2}}+\frac{1}{r_{2}}$ and $1 \leq p \leq q_{1}, q_{2}, r_{1}, r_{2}<\infty$.

Lemma 2.2 (Fratianal chain rule, [9]). Let $G \in C^{1}(\mathbb{C})$ and $s \in(0,1]$, then for any Schwartz function $u$ we have

$$
\left\||\nabla|^{s} G(u)\right\|_{L^{p}\left(\mathbb{R}^{4}\right)} \lesssim\left\|G^{\prime}(u)\right\|_{L^{q}\left(\mathbb{R}^{4}\right)}\left\||\nabla|^{s} u\right\|_{L^{r}\left(\mathbb{R}^{4}\right)},
$$

where $\frac{1}{p}=\frac{1}{q}+\frac{1}{r}$ and $1 \leq p \leq q, r<\infty$.

Now, we are ready to introduce the Strichartz estimates and give the local wellposedness of (CNLS). First, we say a pair of exponents $(q, r)$ is Schrödinger $\dot{H}^{s}$ admissible in dimension four if

$$
\frac{2}{q}=4\left(\frac{1}{2}-\frac{1}{r}\right)-s
$$

and $2 \leq q, r \leq \infty$. And we denote the dual exponent $q^{\prime}$ to $q \in(1, \infty)$ by $\frac{1}{q^{\prime}}+\frac{1}{q}=1$.

Lemma 2.3 (Strichartz estimates, [15, 17, 28]). Let $(q, r)$ and $(\widetilde{q}, \widetilde{r})$ be Schrödinger $L^{2}$-admissible pairs in dimension four. If $\varphi \in L^{2}\left(\mathbb{R}^{4}\right)$ and $f \in L_{t}^{\widetilde{q}^{\prime}} L_{x}^{\widetilde{r}^{\prime}}\left(\mathbb{R} \times \mathbb{R}^{4}\right)$, then we have

$$
\begin{aligned}
&\left\|e^{i t \Delta} \varphi(x)\right\|_{L_{t}^{q} L_{x}^{r}\left(\mathbb{R} \times \mathbb{R}^{4}\right)} \lesssim\|\varphi\|_{L^{2}\left(\mathbb{R}^{4}\right) \cdot} \\
&\left\|\int_{0}^{t} e^{i(t-\tau) \Delta} f(\tau, x) d \tau\right\|_{L_{t}^{q} L_{x}^{r}\left(\mathbb{R} \times \mathbb{R}^{4}\right)} \lesssim\|f\|_{L_{t}^{\tilde{q}^{\prime}} L_{x}^{\tilde{r}^{\prime}}\left(\mathbb{R} \times \mathbb{R}^{4}\right)} .
\end{aligned}
$$

If $I \subseteq \mathbb{R}$ is a interval, we define some time-spatial Strichartz spaces by

$$
\begin{aligned}
& S(I)=L_{t}^{\infty}\left(I ; L^{2}\left(\mathbb{R}^{4}\right)\right) \cap L_{t}^{2}\left(I ; L^{4}\left(\mathbb{R}^{4}\right)\right), \\
& W_{1}(I)=L_{t}^{6}\left(I ; L^{6}\left(\mathbb{R}^{4}\right)\right), \quad V_{1}(I)=L_{t}^{6}\left(I ; L^{\frac{12}{5}}\left(\mathbb{R}^{4}\right)\right), \\
& W_{2}(I)=L_{t}^{4}\left(I ; L^{4}\left(\mathbb{R}^{4}\right)\right), \quad V_{2}(I)=L_{t}^{4}\left(I ; L^{\frac{8}{3}}\left(\mathbb{R}^{4}\right)\right), \\
& S T(I)=W_{1}(I) \cap W_{2}(I), \quad V_{0}(I)=L_{t}^{3}\left(I ; L^{3}\left(\mathbb{R}^{4}\right)\right) .
\end{aligned}
$$

By the definition of the Schrödinger admissible pairs, one can find that $W_{1}$ is $\dot{H}^{1}$ admissible, $W_{2}$ is $\dot{H}^{\frac{1}{2}}$-admissible and $V_{0}, V_{1}, V_{2}$ is $L^{2}$-admissible. On the other hand, standard arguments show that if a solution $u$ to (CNLS) is global, with 
$\|u\|_{S T(\mathbb{R})}<+\infty$, then it scatters. In view of this, we define $\|u\|_{S T(I)}$ as the scattering size of $u$ on time interval $I$ if $u$ is a solution to Cauchy problem (CNLS).

For the sake of later use, by the Hölder inequality and Lemma 2.2. we give some nonlinear estimates:

$$
\left\||\nabla|^{s}\left(|u|^{2} u\right)\right\|_{L_{t, x}^{3 / 2}\left(I \times \mathbb{R}^{4}\right)} \leq\left\||\nabla|^{s} u\right\|_{V_{0}(I)}\|u\|_{W_{1}(I)}^{2}, \quad \text { for } s=0,1
$$

and

$$
\left\||\nabla|^{s}\left(|u|^{\frac{4}{3}} u\right)\right\|_{L_{t, x}^{3 / 2}\left(I \times \mathbb{R}^{4}\right)} \leq\left\||\nabla|^{s} u\right\|_{V_{0}(I)}\|u\|_{W_{2}(I)}^{\frac{4}{3}}, \quad \text { for } s=0,1 .
$$

As a consequence of the Strichartz estimates and the nonlinear estimates, one can obtain the local theory of (CNLS).

Theorem 2.4 (Local well-posedness, 3 ). Let $u_{0} \in H^{1}\left(\mathbb{R}^{4}\right)$ and I be a time interval with $0 \in I$. We have:

(1) There exists $\delta=\delta\left(\left\|u_{0}\right\|_{H^{1}\left(\mathbb{R}^{4}\right)}\right)>0$ such that: If

$$
\left\|\langle\nabla\rangle e^{i t \Delta} u_{0}\right\|_{V_{2}(I)} \leq \delta
$$

then there exists a solution $u \in C\left(I ; H^{1}\left(\mathbb{R}^{4}\right)\right)$ to the Cauchy problem (CNLS) with the following properties:

$$
\begin{aligned}
u(0) & =u_{0}, \\
\|\langle\nabla\rangle u\|_{S(I)} & \leq\left\|u_{0}\right\|_{H^{1}}, \\
\|\langle\nabla\rangle u\|_{V_{2}(I)} & \leq 2\left\|\langle\nabla\rangle e^{i t \Delta} u_{0}\right\|_{V_{2}(I)} .
\end{aligned}
$$

Furthermore, assume that $u \in C\left(I_{\max } ; H^{1}\left(\mathbb{R}^{4}\right)\right)$ is a solution to (CNLS), where $I_{\max }$ is the maximal lifespan of $u$. Note that $I_{\max }$ must be open by (1).

(2) The mass and energy conservation laws hold true, $\forall t, t_{0} \in I_{\max }$,

$$
\begin{aligned}
M(u(t)) & =M\left(u\left(t_{0}\right)\right), \\
E(u(t)) & =E\left(u\left(t_{0}\right)\right) .
\end{aligned}
$$

(3) Let $T_{\max }=\sup I_{\max }$. If $T_{\max }<+\infty$, then

$$
\|u\|_{S T\left(\left[T, T_{\max }\right)\right)}=\infty \text { for any } T \in I_{\max } .
$$

A similar result holds when $T_{\min }=\inf I_{\max }>-\infty$.

(4) If $\|u\|_{S T\left(I_{\max }\right)}<\infty$, then $I_{\max }=\mathbb{R}$ and there exist $\phi_{ \pm} \in H^{1}\left(\mathbb{R}^{4}\right)$ such that

$$
\lim _{t \rightarrow \pm \infty}\left\|u(t)-e^{i t \Delta} \phi_{ \pm}\right\|_{H^{1}\left(\mathbb{R}^{4}\right)}=0 .
$$

Similar to [3, Proposition 5.6], we have the Perturbation theory.

Proposition 2.5 (Perturbation theory, [3]). Let I be an interval, $u \in C\left(I ; H^{1}\left(\mathbb{R}^{4}\right)\right)$ be a solution to (CNLS) and $\tilde{u}$ be a function in $C\left(I ; H^{1}\left(\mathbb{R}^{4}\right)\right)$. Let $A>0$ and $t_{1} \in I$ such that

$$
\|u\|_{L^{\infty}\left(I ; H^{1}\right)}+\left\|u\left(t_{1}\right)-\tilde{u}\left(t_{1}\right)\right\|_{H^{1}}+\|\tilde{u}\|_{S T(I)} \leq A .
$$

Then there exists $\delta>0$ depending on $A$ such that if

$$
\begin{array}{r}
\left\|\langle\nabla\rangle\left(\left(i \partial_{t}+\Delta\right) \tilde{u}+|\tilde{u}|^{2} \tilde{u}-|\tilde{u}|^{\frac{4}{3}} \tilde{u}\right)\right\|_{L^{\frac{3}{2}}\left(I \times \mathbb{R}^{4}\right)} \leq \delta, \\
\left\|\langle\nabla\rangle e^{i\left(t-t_{1}\right) \Delta}\left[u\left(t_{1}\right)-\tilde{u}\left(t_{1}\right)\right]\right\|_{V_{2}(I)} \leq \delta .
\end{array}
$$

Then we have $\|\langle\nabla\rangle u\|_{S(I)}<\infty$. 
2.2. Variational methods. First we recall the energy of the solution $u$ to (CNLS)

$$
E(u)(t)=\frac{1}{2} \int|\nabla u(t, x)|^{2} d x-\frac{1}{4} \int|u(t, x)|^{4} d x+\frac{3}{10} \int|u(t, x)|^{\frac{10}{3}} d x,
$$

and the modified energy

$$
E_{c}(u)(t)=\frac{1}{2} \int|\nabla u(t, x)|^{2} d x-\frac{1}{4} \int|u(t, x)|^{4} d x .
$$

Recall in the introduction, for any functional $F$ of $H^{1}$, we define its variation differential by

$$
\mathcal{L}_{\alpha, \beta} F(\phi)=\left.\frac{d}{d \lambda}\right|_{\lambda=0} F\left(\phi_{\alpha, \beta}^{\lambda}\right)=F([(\alpha-\beta x \cdot \nabla) \phi](x))
$$

where $\phi_{\alpha, \beta}^{\lambda}(x)=e^{\alpha \lambda} \phi\left(e^{-\beta \lambda} x\right)$, if $(\alpha, \beta, \lambda) \in \mathbb{R}^{3}$. Thus we have

$K_{\alpha, \beta}(\phi):=\mathcal{L}_{\alpha, \beta} E(\phi)=(\alpha+\beta) \int\left[|\nabla \phi(x)|^{2}-|\phi(x)|^{4}\right] d x+\left(\alpha+\frac{6}{5} \beta\right) \int|\phi(x)|^{\frac{10}{3}} d x$.

By the definition of the region of $(\alpha, \beta)$,

$$
\Omega=\{(\alpha, \beta): \alpha \geq 0,5 \alpha+6 \beta \geq 0,(\alpha, \beta) \neq(0,0)\},
$$

it is easy to check that $\alpha+\beta>0$ if $(\alpha, \beta) \in \Omega$.

Proposition 2.6. For any $(\alpha, \beta) \in \Omega$, if $\left\{\varphi_{n}\right\}_{n \geq 1}$ is a sequence in $H^{1}\left(\mathbb{R}^{4}\right)$ with

$$
\lim _{n \rightarrow \infty}\left\|\varphi_{n}\right\|_{\dot{H}^{1}}=0
$$

then, we have

$$
K_{\alpha, \beta}\left(\varphi_{n}\right)>0
$$

for sufficient large $n$.

Proof. From the Sobolev inequalities

$$
\left\|\varphi_{n}\right\|_{L^{4}\left(\mathbb{R}^{4}\right)} \lesssim\left\|\varphi_{n}\right\|_{\dot{H}^{1}\left(\mathbb{R}^{4}\right)},
$$

we have $\left\|\varphi_{n}\right\|_{L^{4}\left(\mathbb{R}^{4}\right)}^{4}=o\left(\left\|\varphi_{n}\right\|_{\dot{H}^{1}\left(\mathbb{R}^{4}\right)}^{2}\right)$ as $n \rightarrow \infty$, which together with (2.20) implies (2.21).

We define $\bar{\mu}$ by

$$
\bar{\mu}=\max \left\{2(\alpha+\beta), \frac{10}{3} \alpha+4 \beta\right\}=\left\{\begin{array}{l}
2(\alpha+\beta) \quad \text { if } \quad 2 \alpha \leq-3 \beta \\
\frac{10}{3} \alpha+4 \beta \quad \text { if } \quad 2 \alpha \geq-3 \beta .
\end{array}\right.
$$

By a direct calculation, we have

\section{Lemma 2.7.}

$$
\left(\bar{\mu}-\mathcal{L}_{\alpha, \beta}\right) E(\varphi)=\left\{\begin{array}{l}
\frac{\alpha+\beta}{2}\|\varphi\|_{L^{4}}^{4}-\frac{2 \alpha+3 \beta}{5}\|\varphi\|_{L^{\frac{10}{3}}}^{\frac{10}{3}} \quad \text { if } \quad 2 \alpha \leq-3 \beta \\
\left(\frac{2}{3} \alpha+\beta\right)\|\varphi\|_{\dot{H}^{1}}^{2}+\frac{1}{6} \alpha\|\varphi\|_{L^{4}}^{4} \quad \text { if } \quad 2 \alpha \geq-3 \beta
\end{array}\right.
$$

and

$$
\begin{aligned}
& \mathcal{L}_{\alpha, \beta}\left(\bar{\mu}-\mathcal{L}_{\alpha, \beta}\right) E(\varphi) \\
= & \left\{\begin{array}{l}
2(\alpha+\beta)^{2}\|\varphi\|_{L^{4}}^{4}-\frac{2}{15}(2 \alpha+3 \beta)(5 \alpha+6 \beta)\|\varphi\|_{L^{\frac{10}{3}}}^{\frac{10}{3}} \quad \text { if } \quad 2 \alpha \leq-3 \beta, \\
\left(\frac{2}{3} \alpha+\beta\right)(2 \alpha+2 \beta)\|\varphi\|_{\dot{H}^{1}}^{2}+\frac{2}{3} \alpha(\alpha+\beta)\|\varphi\|_{L^{4}}^{4} \quad \text { if } \quad 2 \alpha \geq-3 \beta .
\end{array}\right.
\end{aligned}
$$


This lemma impies that $H_{\alpha, \beta}(\varphi)>0$ and $\mathcal{L}_{\alpha, \beta} H_{\alpha, \beta}(\varphi)>0$ for $\varphi \in H^{1}\left(\mathbb{R}^{4}\right) \backslash\{0\}$, where $H_{\alpha, \beta}$ is given by

$$
\begin{aligned}
H_{\alpha, \beta}(\varphi) & =\left(1-\frac{\mathcal{L}_{\alpha, \beta}}{\bar{\mu}}\right) E(\varphi) \\
& =\left\{\begin{array}{l}
\frac{1}{4}\|\varphi\|_{L^{4}}^{4}-\frac{2 \alpha+3 \beta}{10(\alpha+\beta)}\|\varphi\|_{L^{\frac{10}{3}}}^{\frac{10}{3}} \text { if } 2 \alpha \leq-3 \beta \\
\frac{2 \alpha+3 \beta}{10 \alpha+12 \beta}\|\varphi\|_{\dot{H}^{1}}^{2}+\frac{\alpha}{20 \alpha+24 \beta}\|\varphi\|_{L^{4}}^{4} \quad \text { if } \quad 2 \alpha \geq-3 \beta
\end{array}\right.
\end{aligned}
$$

As in the introduction, we define

$$
m_{\alpha, \beta}=\inf \left\{E(\varphi): \varphi \in H^{1}\left(\mathbb{R}^{4}\right) \backslash\{0\}, K_{\alpha, \beta}(\varphi)=0\right\} .
$$

Lemma 2.8. For $(\alpha, \beta) \in \Omega$, we have

$$
\begin{aligned}
m_{\alpha, \beta} & =\inf \left\{H_{\alpha, \beta}(\varphi): \varphi \in H^{1}\left(\mathbb{R}^{4}\right) \backslash\{0\}, K_{\alpha, \beta}(\varphi) \leq 0\right\} \\
& =\inf \left\{H_{\alpha, \beta}(\varphi): \varphi \in H^{1}\left(\mathbb{R}^{4}\right) \backslash\{0\}, K_{\alpha, \beta}(\varphi)<0\right\} .
\end{aligned}
$$

Proof. Let $m_{\alpha, \beta}^{\prime}=\inf \left\{H_{\alpha, \beta}(\varphi): \varphi \in H^{1}\left(\mathbb{R}^{4}\right) \backslash\{0\}, K_{\alpha, \beta}(\varphi) \leq 0\right\}$ and

$$
m_{\alpha, \beta}^{\prime \prime}=\inf \left\{H_{\alpha, \beta}(\varphi): \varphi \in H^{1}\left(\mathbb{R}^{4}\right) \backslash\{0\}, K_{\alpha, \beta}(\varphi)<0\right\} .
$$

If $K_{\alpha, \beta}(\varphi)=0$, then we have $H_{\alpha, \beta}(\varphi)=E(\varphi)$. Hence we have

$$
m_{\alpha, \beta} \geq m_{\alpha, \beta}^{\prime} \text {. }
$$

On the other hand, if $K_{\alpha, \beta}(\varphi)<0$, from Proposition 2.6, there exists $\lambda_{0}<0$ such that

$$
K_{\alpha, \beta}\left(\varphi_{\alpha, \beta}^{\lambda_{0}}\right)=0
$$

By Lemma 2.7. we have $\mathcal{L}_{\alpha, \beta} H_{\alpha, \beta}(\phi)>0$ for any $\phi \in H^{1}$, which implies

$$
H_{\alpha, \beta}\left(\varphi_{\alpha, \beta}^{\lambda_{0}}\right)<H_{\alpha, \beta}(\varphi) \text {. }
$$

This implies $m_{\alpha, \beta} \leq m_{\alpha, \beta}^{\prime}$, which together with (2.26) implies $m_{\alpha, \beta}=m_{\alpha, \beta}^{\prime}$. For the second equality in (2.25), one can easily find that

$$
m_{\alpha, \beta}^{\prime} \leq m_{\alpha, \beta}^{\prime \prime} .
$$

For any $\varphi \in H^{1}\left(\mathbb{R}^{4}\right) \backslash\{0\}$ such that $K_{\alpha, \beta}(\varphi) \leq 0$, from Lemma 2.7 we have

$$
\mathcal{L}_{\alpha, \beta} K_{\alpha, \beta}(\varphi)=\bar{\mu} K_{\alpha, \beta}(\varphi)-H_{\alpha, \beta}(\varphi)<0 .
$$

This implies for $\lambda>0, K_{\alpha, \beta}\left(\varphi_{\alpha, \beta}^{\lambda}\right)<0$. And by definition of $H_{\alpha, \beta}$, we have as $\lambda \rightarrow 0$,

which implies $m_{\alpha, \beta}^{\prime} \geq m_{\alpha, \beta}^{\prime \prime}$.

$$
H_{\alpha, \beta}\left(\varphi_{\alpha, \beta}^{\lambda}\right) \rightarrow H_{\alpha, \beta}(\varphi)
$$

Next we will use the ( $\dot{H}^{1}$-invariant) scaling argument to remove the $L^{\frac{10}{3}}$-term (the lower regularity quantity than $\dot{H}^{1}$ ) in $K_{\alpha, \beta}$, that is, to replace the constrained condition $K_{\alpha, \beta}(\varphi)<0$ by $K_{\alpha, \beta}^{c}(\varphi)<0$, where

$$
K_{\alpha, \beta}^{c}(\varphi)=\mathcal{L}_{\alpha, \beta} E_{c}(\varphi)=(\alpha+\beta) \int|\nabla \varphi(x)|^{2} d x-(\alpha+\beta) \int|\varphi(x)|^{4} d x .
$$

And let

$$
H_{\alpha, \beta}^{c}(\varphi)=\left(1-\frac{\mathcal{L}_{\alpha, \beta}}{\bar{\mu}}\right) E_{c}(\varphi)=\left\{\begin{array}{l}
\frac{1}{4}\|\varphi\|_{L^{4}}^{4} \quad \text { if } 2 \alpha \leq-3 \beta, \\
\frac{2 \alpha+3 \beta}{10 \alpha+12 \beta}\|\varphi\|_{\dot{H}^{1}}^{2}+\frac{\alpha}{20 \alpha+24 \beta}\|\varphi\|_{L^{4}}^{4} \quad \text { if } \quad 2 \alpha \geq-3 \beta .
\end{array}\right.
$$


Lemma 2.9. For $(\alpha, \beta) \in \Omega$, we have

$$
\begin{aligned}
m_{\alpha, \beta} & =\inf \left\{H_{\alpha, \beta}(\varphi): \varphi \in H^{1}\left(\mathbb{R}^{4}\right) \backslash\{0\}, K_{\alpha, \beta}^{c}(\varphi) \leq 0\right\} \\
& =\inf \left\{H_{\alpha, \beta}(\varphi): \varphi \in H^{1}\left(\mathbb{R}^{4}\right) \backslash\{0\}, K_{\alpha, \beta}^{c}(\varphi)<0\right\} \\
& =\inf \left\{H_{\alpha, \beta}^{c}(\varphi): \varphi \in H^{1}\left(\mathbb{R}^{4}\right) \backslash\{0\}, K_{\alpha, \beta}^{c}(\varphi)<0\right\} \\
& =\inf \left\{H_{\alpha, \beta}^{c}(\varphi): \varphi \in H^{1}\left(\mathbb{R}^{4}\right) \backslash\{0\}, K_{\alpha, \beta}^{c}(\varphi) \leq 0\right\} \\
& =\inf \left\{H_{\alpha, \beta}^{c}(\varphi): \varphi \in H^{1}\left(\mathbb{R}^{4}\right) \backslash\{0\}, K_{\alpha, \beta}^{c}(\varphi)=0\right\} .
\end{aligned}
$$

Proof. First, we denote $m_{\alpha, \beta}^{(1)}$ and $m_{\alpha, \beta}^{(2)}$ by

$$
m_{\alpha, \beta}^{(1)}=\inf \left\{H_{\alpha, \beta}(\varphi): \varphi \in H^{1}\left(\mathbb{R}^{4}\right) \backslash\{0\}, K_{\alpha, \beta}^{c}(\varphi) \leq 0\right\}
$$

and

$$
m_{\alpha, \beta}^{(2)}=\inf \left\{H_{\alpha, \beta}(\varphi): \varphi \in H^{1}\left(\mathbb{R}^{4}\right) \backslash\{0\}, K_{\alpha, \beta}^{c}(\varphi)<0\right\} .
$$

From the definition of $H_{\alpha, \beta}$ and the fact that $K_{\alpha, \beta}(\varphi) \geq K_{\alpha, \beta}^{c}(\varphi)$, we have

$$
m_{\alpha, \beta}^{(2)} \leq m_{\alpha, \beta}^{\prime \prime}=m_{\alpha, \beta}
$$

To prove $m_{\alpha, \beta}^{(2)} \geq m_{\alpha, \beta}^{\prime \prime}$, for any $\varphi \in H^{1}\left(\mathbb{R}^{4}\right) \backslash\{0\}$ with $K_{\alpha, \beta}^{c}(\varphi)<0$, we have

$$
K_{\alpha, \beta}\left(\varphi_{1,-1}^{\lambda}\right)=(\alpha+\beta)\left(\|\nabla \varphi\|_{L^{2}}^{2}-\|\varphi\|_{L^{4}}^{4}\right)+e^{-\frac{2}{3} \lambda}\left(\alpha+\frac{6}{5} \beta\right)\|\varphi\|_{L^{\frac{10}{3}}}^{\frac{10}{3}} \rightarrow K_{\alpha, \beta}^{c}(\varphi),
$$

as $\lambda \rightarrow+\infty$. And for $\lambda>0, H_{\alpha, \beta}\left(\varphi_{1,-1}^{\lambda}\right) \leq H_{\alpha, \beta}(\varphi)$, which implies $m_{\alpha, \beta}^{(2)} \geq m_{\alpha, \beta}^{\prime \prime}$. On the other hand, it is trivial that

$$
m_{\alpha, \beta}^{(1)} \leq m_{\alpha, \beta}^{(2)} .
$$

Next, we need to prove $m_{\alpha, \beta}^{(2)} \leq m_{\alpha, \beta}^{(1)}$. Let $\varphi \in H^{1}\left(\mathbb{R}^{4}\right) \backslash\{0\}$ such that $K_{\alpha, \beta}^{c}(\varphi) \leq 0$, then from

$$
\mathcal{L}_{\alpha, \beta} K_{\alpha, \beta}^{c}(\varphi)=2(\alpha+\beta)^{2}\left(\|\nabla \varphi\|_{L^{2}}^{2}-2\|\varphi\|_{L^{4}}^{4}\right)<0,
$$

we have $K_{\alpha, \beta}^{c}\left(\varphi_{\alpha, \beta}^{\lambda}\right)<0$, for $\lambda>0$. As above we have $H_{\alpha, \beta}\left(\varphi_{\alpha, \beta}^{\lambda}\right) \rightarrow H_{\alpha, \beta}(\varphi)$, as $\lambda \rightarrow 0$. This implies $m_{\alpha, \beta}^{(2)} \leq m_{\alpha, \beta}^{(1)}$.

Second, we denote

$$
m_{\alpha, \beta}^{c}=\inf \left\{H_{\alpha, \beta}^{c}(\varphi): \varphi \in H^{1}\left(\mathbb{R}^{4}\right) \backslash\{0\}, K_{\alpha, \beta}^{c}(\varphi)=0\right\} .
$$

One can easily find that

$$
\begin{aligned}
& \inf \left\{H_{\alpha, \beta}^{c}(\varphi): \varphi \in H^{1}\left(\mathbb{R}^{4}\right) \backslash\{0\}, K_{\alpha, \beta}^{c}(\varphi) \leq 0\right\} \\
\leq & \inf \left\{H_{\alpha, \beta}^{c}(\varphi): \varphi \in H^{1}\left(\mathbb{R}^{4}\right) \backslash\{0\}, K_{\alpha, \beta}^{c}(\varphi)<0\right\} .
\end{aligned}
$$

Let $\varphi \in H^{1}\left(\mathbb{R}^{4}\right) \backslash\{0\}$ such that $K_{\alpha, \beta}^{c}(\varphi) \leq 0$. For $\lambda>0$, we have $K_{\alpha, \beta}^{c}\left(\varphi_{\alpha, \beta}^{\lambda}\right)<0$, and

Hence, we have

$$
H_{\alpha, \beta}^{c}\left(\varphi_{\alpha, \beta}^{\lambda}\right) \rightarrow H_{\alpha, \beta}^{c}(\varphi), \text { as } \lambda \rightarrow 0
$$

$$
\begin{aligned}
& \inf \left\{H_{\alpha, \beta}^{c}(\varphi): \varphi \in H^{1}\left(\mathbb{R}^{4}\right) \backslash\{0\}, K_{\alpha, \beta}^{c}(\varphi) \leq 0\right\} \\
\geq & \inf \left\{H_{\alpha, \beta}^{c}(\varphi): \varphi \in H^{1}\left(\mathbb{R}^{4}\right) \backslash\{0\}, K_{\alpha, \beta}^{c}(\varphi)<0\right\} .
\end{aligned}
$$

It is trivial that

$$
\inf \left\{H_{\alpha, \beta}^{c}(\varphi): \varphi \in H^{1}\left(\mathbb{R}^{4}\right) \backslash\{0\}, K_{\alpha, \beta}^{c}(\varphi) \leq 0\right\} \leq m_{\alpha, \beta}^{c} .
$$


Let $\varphi \in H^{1}\left(\mathbb{R}^{4}\right) \backslash\{0\}$ such that $K_{\alpha, \beta}^{c}(\varphi)<0$. Then there exist $\lambda_{0}<0$ such that

$$
K_{\alpha, \beta}^{c}\left(\varphi_{\alpha, \beta}^{\lambda_{0}}\right)=0
$$

From $\mathcal{L}_{\alpha, \beta} H_{\alpha, \beta}(\phi)>0$ for any $\phi \in H^{1}\left(\mathbb{R}^{4}\right) \backslash\{0\}$, we have $H_{\alpha, \beta}^{c}\left(\varphi_{\alpha, \beta}^{\lambda_{0}}\right) \leq H_{\alpha, \beta}^{c}(\varphi)$, which implies

$$
\inf \left\{H_{\alpha, \beta}^{c}(\varphi): \varphi \in H^{1}\left(\mathbb{R}^{4}\right) \backslash\{0\}, K_{\alpha, \beta}^{c}(\varphi) \leq 0\right\} \geq m_{\alpha, \beta}^{c}
$$

Combine (2.31), (2.32), (2.33) (2.34) (2.35) together, we can obtain the last two inequalities in (2.28).

Finally, we just need to show $m_{\alpha, \beta}^{c}=m_{\alpha, \beta}$. It is trivial that $m_{\alpha, \beta}^{c} \leq m_{\alpha, \beta}$. By the definition of $H_{\alpha, \beta}^{c}$ and $H_{\alpha, \beta}$, we just need to show $m_{\alpha, \beta}^{c} \geq m_{\alpha, \beta}$ in the case of $2 \alpha<-3 \beta$.

Let $\phi \in H^{1}\left(\mathbb{R}^{4}\right) \backslash\{0\}$ such that $K_{\alpha, \beta}^{c}(\varphi)<0$. Then for any $\lambda \in \mathbb{R}, K_{\alpha, \beta}^{c}\left(\varphi_{1,-1}^{\lambda}\right)=$ $K_{\alpha, \beta}^{c}(\varphi)$, and

$$
H_{\alpha, \beta}\left(\varphi_{1,-1}^{\lambda}\right)=H_{\alpha, \beta}^{c}(\varphi)-\frac{2 \alpha+3 \beta}{10(\alpha+\beta)} e^{-\frac{2}{3} \lambda}\|\varphi\|_{L^{\frac{10}{3}}}^{\frac{10}{3}} \rightarrow H_{\alpha, \beta}^{c}(\varphi) \text { as } \lambda \rightarrow+\infty .
$$

This implies $m_{\alpha, \beta}^{c} \geq m_{\alpha, \beta}$.

Therefore, we complete the proof of Lemma 2.9.

We remark that by Lemma 2.9 and the definition of $m_{\alpha, \beta}^{c}$ and $H_{\alpha, \beta}^{c}$, we have

$$
m_{\alpha, \beta}=m_{\alpha, \beta}^{c}=\inf \left\{E_{c}(\varphi): \varphi \in H^{1}\left(\mathbb{R}^{4}\right) \backslash\{0\},\|\nabla \varphi\|_{L^{2}}^{2}=\|\varphi\|_{L^{4}}^{4}\right\} .
$$

which implies that $m_{\alpha, \beta}$ is independent of $(\alpha, \beta)$ if $(\alpha, \beta) \in \Omega$. Hence we can denote $m$ by $m=m_{\alpha, \beta}$ for any $(\alpha, \beta) \in \Omega$. Now, we can make use of the sharp Sobolev constant in [1, 29] to compute the minimization $m$, which also shows Proposition 1.3 .

Lemma 2.10. For the minimization $m_{\alpha, \beta}$, we have

$$
m=E_{c}(W)
$$

Proof. From Lemma 2.9 and (2.36), we have

$$
\begin{aligned}
m & =\inf \left\{E_{c}(\varphi): \varphi \in H^{1}\left(\mathbb{R}^{4}\right) \backslash\{0\},\|\nabla \varphi\|_{L^{2}}^{2}=\|\varphi\|_{L^{4}}^{4}\right\} \\
& =\inf \left\{\frac{1}{4}\|\nabla \varphi\|_{L^{2}}^{2}: \varphi \in H^{1}\left(\mathbb{R}^{4}\right) \backslash\{0\},\|\nabla \varphi\|_{L^{2}}^{2}=\|\varphi\|_{L^{4}}^{4}\right\} \\
& \geq \inf \left\{\frac{1}{4}\|\nabla \varphi\|_{L^{2}}^{2}: \varphi \in H^{1}\left(\mathbb{R}^{4}\right) \backslash\{0\},\|\nabla \varphi\|_{L^{2}}^{2} \leq\|\varphi\|_{L^{4}}^{4}\right\},
\end{aligned}
$$


where the equality holds if and only if the minimization is attained by some $\varphi$ with $\|\nabla \varphi\|_{L^{2}}^{2}=\|\varphi\|_{L^{4}}^{4}$. Next, we have

$$
\begin{aligned}
& \inf \left\{\frac{1}{4}\|\nabla \varphi\|_{L^{2}}^{2}: \varphi \in H^{1}\left(\mathbb{R}^{4}\right) \backslash\{0\},\|\nabla \varphi\|_{L^{2}}^{2} \leq\|\varphi\|_{L^{4}}^{4}\right\} \\
= & \inf \left\{\frac{1}{4}\|\nabla \varphi\|_{L^{2}}^{2}: \varphi \in \dot{H}^{1}\left(\mathbb{R}^{4}\right) \backslash\{0\},\|\nabla \varphi\|_{L^{2}}^{2} \leq\|\varphi\|_{L^{4}}^{4}\right\} \\
= & \inf \left\{\frac{1}{4}\left(\frac{\|\nabla \varphi\|_{L^{2}}}{\|\varphi\|_{L^{4}}}\right)^{4}: \varphi \in \dot{H}^{1}\left(\mathbb{R}^{4}\right) \backslash\{0\},\|\nabla \varphi\|_{L^{2}}^{2} \leq\|\varphi\|_{L^{4}}^{4}\right\} \\
= & \frac{1}{4}\|W\|_{L^{4}}^{4} \\
= & E_{c}(W),
\end{aligned}
$$

where we used the fact that $H^{1}$ is dense in $\dot{H}^{1}$ and the sharp Sobolev inequality

$$
\|\varphi\|_{L^{4}\left(\mathbb{R}^{4}\right)} \leq\|W\|_{L^{4}\left(\mathbb{R}^{4}\right)}^{-1}\|\nabla \varphi\|_{L^{2}\left(\mathbb{R}^{4}\right)} .
$$

Hence we can define $\mathcal{K}_{\alpha, \beta}^{+}$and $\mathcal{K}_{\alpha, \beta}^{-}$by

$$
\mathcal{K}_{\alpha, \beta}^{+}=\left\{\varphi \in H^{1}\left(\mathbb{R}^{4}\right): E(\varphi)<m, K_{\alpha, \beta}(\varphi) \geq 0\right\}
$$

and

$$
\mathcal{K}_{\alpha, \beta}^{-}=\left\{\varphi \in H^{1}\left(\mathbb{R}^{4}\right): E(\varphi)<m, K_{\alpha, \beta}(\varphi)<0\right\} .
$$

Now we are going to prove that the regions $\mathcal{K}_{\alpha, \beta}^{+}$and $\mathcal{K}_{\alpha, \beta}^{-}$are independent of $(\alpha, \beta)$. The proof is mainly based on the minimal property of $m_{\alpha, \beta}$ and the relation between energy $E$ and its variational derivatives $K_{\alpha, \beta}$, which is similar to Lemma 2.9 in 16 .

Lemma 2.11. If $(\alpha, \beta) \in \Omega$, then $\mathcal{K}_{\alpha, \beta}^{ \pm}$are independent of $(\alpha, \beta)$.

Proof. From the definition of $\Omega$ in (2.20), we have $\alpha+\beta>0$, if $(\alpha, \beta) \in \Omega$. First, $\mathcal{K}_{\alpha, \beta}^{+} \cup \mathcal{K}_{\alpha, \beta}^{-}$is independent of $(\alpha, \beta)$, since

$$
\mathcal{K}_{\alpha, \beta}^{+} \cup \mathcal{K}_{\alpha, \beta}^{-}=\left\{\varphi \in H^{1}\left(\mathbb{R}^{4}\right): E(\varphi)<m\right\} .
$$

For $\varphi \in \mathcal{K}_{\alpha, \beta}^{+}$, by the definition of $m$, if $K_{\alpha, \beta}(\varphi)=0$, then $\varphi=0$. Hence if $\varphi \in \mathcal{K}_{\alpha, \beta}^{+}$ and $\varphi \neq 0$, then we have $K_{\alpha, \beta}(\varphi)>0$. From this we have the scaling variation of $\varphi \in \mathcal{K}_{\alpha, \beta}^{+}, \varphi_{\alpha, \beta}^{\lambda}$ such that the following properties:

(1) By the definition of $m, K_{\alpha, \beta}\left(\varphi_{\alpha, \beta}^{\lambda}\right)>0$ is preserved, provided $E\left(\varphi_{\alpha, \beta}^{\lambda}\right)<m$;

(2) $E\left(\varphi_{\alpha, \beta}^{\lambda}\right)$ does not increase, as $\lambda \leq 0$ decreases, if

$$
\mathcal{L}_{\alpha, \beta} E\left(\varphi_{\alpha, \beta}^{\lambda}\right)=K_{\alpha, \beta}\left(\varphi_{\alpha, \beta}^{\lambda}\right)>0 .
$$

If there exists $\varphi \in \mathcal{K}_{\alpha, \beta}^{+} \cap \mathcal{K}_{\alpha^{\prime}, \beta^{\prime}}^{-}$, where $(\alpha, \beta),\left(\alpha^{\prime}, \beta^{\prime}\right) \in \Omega$. By the definition of $m$ and the fact that $m>0$, we have $\varphi \neq 0$ and $K_{\alpha, \beta}(\varphi)>0$.

Then, from the above two facts, we have $E\left(\varphi_{\alpha, \beta}^{\lambda}\right)<m$, for $\lambda \leq 0$. And by $\alpha+\beta>0$, we have $\varphi_{\alpha, \beta}^{\lambda} \rightarrow 0$ in $\dot{H}^{1}$, as $\lambda \rightarrow-\infty$. But by Proposition 2.6, there exists $\lambda_{0}<0$ such that $K_{\alpha^{\prime}, \beta^{\prime}}\left(\varphi_{\alpha, \beta}^{\lambda_{0}}\right)=0$, which together with $E\left(\varphi_{\alpha, \beta}^{\lambda_{0}}\right)<m$ contradicts the minimization of $m$. Hence $\mathcal{K}_{\alpha, \beta}^{+} \cap \mathcal{K}_{\alpha^{\prime}, \beta^{\prime}}^{-}=\emptyset$ for any $(\alpha, \beta),\left(\alpha^{\prime}, \beta^{\prime}\right) \in \Omega$, which and (2.41) imply the claim. 
After the computation of the minimization $m$, we next give some variational estimates.

Lemma 2.12. For any $\varphi \in H^{1}\left(\mathbb{R}^{4}\right)$ with $K_{2,-1}(\varphi) \geq 0$, we have

$$
\int_{\mathbb{R}^{4}}\left(\frac{1}{4}|\nabla \varphi|^{2}+\frac{1}{10}|\varphi|^{\frac{10}{3}}\right) \leq E(\varphi) \leq \int_{\mathbb{R}^{4}}\left(\frac{1}{2}|\nabla \varphi|^{2}+\frac{3}{10}|\varphi|^{\frac{10}{3}}\right) .
$$

Proof. On one hand, the right hand side of (2.42) is trivial. On the other hand, by the definition of $E$ and $K_{\alpha, \beta}$, we have

$$
E(\varphi)=\int_{\mathbb{R}^{4}}\left(\frac{1}{4}|\nabla \varphi|^{2}+\frac{1}{10}|\varphi|^{\frac{10}{3}}\right)+\frac{1}{4} K_{2,-1}(\varphi),
$$

which implies the left hand side of (2.42).

At the last of this section, we give the uniform bounds on the scaling derivative functional $K_{\alpha, \beta}(\varphi)$ with the energy $E(\varphi)$ below the threshold $m$, which plays an important role for the blow-up and scattering analysis.

Lemma 2.13. For any $\varphi \in H^{1}$ with $E(\varphi)<m$.

(1) If $K_{2,-1}(\varphi)<0$, then

$$
K_{2,-1}(\varphi) \leq-\frac{8}{3}(m-E(\varphi)),
$$

and

$$
\|\nabla \varphi\|_{L_{x}^{2}}>\|\nabla W\|_{L_{x}^{2}}
$$

(2) If $K_{2,-1}(\varphi) \geq 0$, then

$$
\begin{aligned}
& K_{2,-1}(\varphi) \geq \min \left(\frac{8}{3}(m-E(\varphi)), \frac{1}{5}\|\nabla \varphi\|_{L^{2}}^{2}+\frac{1}{100}\|\varphi\|_{L^{\frac{10}{3}}}^{\frac{10}{3}}\right), \\
& \text { and }
\end{aligned}
$$

$$
\|\nabla \varphi\|_{L_{x}^{2}}<\|\nabla W\|_{L_{x}^{2}}
$$

Proof. By Lemma 2.7, for any $\varphi \in H^{1}$, we have

$$
\mathcal{L}_{2,-1}^{2} E(\varphi)=\frac{8}{3} \mathcal{L}_{2,-1} E(\varphi)-\frac{2}{3}\|\nabla \varphi\|_{L^{2}}^{2}-\frac{4}{3}\|\varphi\|_{L^{4}}^{4} .
$$

Let $j(\lambda)=E\left(\varphi_{2,-1}^{\lambda}\right)$, then we have

$$
j^{\prime \prime}(\lambda)=\frac{8}{3} j^{\prime}(\lambda)-\frac{2}{3} e^{2 \lambda}\|\nabla \varphi\|_{L^{2}}^{2}-\frac{4}{3} e^{4 \lambda}\|\varphi\|_{L^{4}}^{4} .
$$

Case I: If $K_{2,-1}(\varphi)<0$, then by

$$
\lim _{\lambda \rightarrow-\infty}\left\|\varphi_{2,-1}^{\lambda}\right\|_{\dot{H}^{1}}^{2}=0
$$

Proposition 2.6 and the continuity of $K_{2,-1}$ in $\lambda$, there exists a negative number $\lambda_{0}<0$ such that $K_{2,-1}\left(\varphi_{2,-1}^{\lambda_{0}}\right)=0$, and

$$
K_{2,-1}\left(\varphi_{2,-1}^{\lambda}\right)<0, \quad \forall \lambda \in\left(\lambda_{0}, 0\right) .
$$


By the definition of $m$, we obtain $j\left(\lambda_{0}\right)=E\left(\varphi_{2,-1}^{\lambda_{0}}\right) \geq m$. Now by integrating (2.47) over $\left[\lambda_{0}, 0\right]$, we have

$$
\int_{\lambda_{0}}^{0} j^{\prime \prime}(\lambda) d \lambda \leq \frac{8}{3} \int_{\lambda_{0}}^{0} j^{\prime}(\lambda) d \lambda
$$

This yields that

$$
K_{2,-1}(\varphi)=j^{\prime}(0)-j^{\prime}\left(\lambda_{0}\right) \leq \frac{8}{3}\left(j(0)-j\left(\lambda_{0}\right)\right) \leq-\frac{8}{3}(m-E(\varphi)),
$$

which implies (2.43).

Since $K_{2,-1}(\varphi)<0$, we have by Lemma 2.8

$$
m \leq H_{2,-1}(\varphi)<\frac{1}{4}\|\varphi\|_{L^{4}}^{4} .
$$

where we have used the fact that $K_{2,-1}(\varphi)<0$ in the second inequality. By the fact $m=\frac{1}{4}\left(C_{4}^{*}\right)^{-4}$ and the Sharp Sobolev inequality, we have

$$
\|\nabla \varphi\|_{L^{2}}^{4} \geq\left(C_{4}^{*}\right)^{-4}\|\varphi\|_{L^{4}}^{4}>(4 m)^{2},
$$

which implies that $\|\nabla \varphi\|_{L^{2}}^{2}>4 m=\|\nabla W\|_{L_{x}^{2}}^{2}$. Thus, we conclude (2.44).

Case II: $K_{2,-1}(\varphi) \geq 0$. We divide it into two subcases:

When $4 K_{2,-1}(\varphi) \geq\|\varphi\|_{L^{4}}^{4}$. Since

$$
\frac{4}{3} \int_{\mathbb{R}^{4}}|\varphi|^{4} d x=-\frac{4}{3} K_{2,-1}(\varphi)+\int_{\mathbb{R}^{4}}\left(\frac{4}{3}|\nabla \varphi|^{2}+\frac{1}{15}|\varphi|^{\frac{10}{3}}\right) d x,
$$

then we have

$$
\frac{16}{3} K_{2,-1}(\varphi) \geq-\frac{4}{3} K_{2,-1}(\varphi)+\int_{\mathbb{R}^{4}}\left(\frac{4}{3}|\nabla \varphi|^{2}+\frac{1}{15}|\varphi|^{\frac{10}{3}}\right) d x,
$$

which implies that

$$
K_{2,-1}(\varphi) \geq \frac{1}{5}\|\nabla \varphi\|_{L^{2}}^{2}+\frac{1}{100}\|\varphi\|_{L^{\frac{10}{3}}}^{\frac{10}{3}} .
$$

When $4 K_{2,-1}(\varphi) \leq\|\varphi\|_{L^{4}}^{4}$. By (2.47), we have for $\lambda=0$

$$
\begin{gathered}
0<\frac{16}{3} j^{\prime}(\lambda)<\frac{4}{3} e^{4 \lambda}\|\varphi\|_{L^{4}}^{4}, \\
j^{\prime \prime}(\lambda)=\frac{8}{3} j^{\prime}(\lambda)-\frac{2}{3} e^{2 \lambda}\|\nabla \varphi\|_{L^{2}}^{2}-\frac{4}{3} e^{4 \lambda}\|\varphi\|_{L^{4}}^{4} \leq-\frac{8}{3} j^{\prime}(\lambda) .
\end{gathered}
$$

By the continuity of $j^{\prime}$ and $j^{\prime \prime}$ in $\lambda$, we know that $j^{\prime}$ is an accelerating decreasing function as $\lambda$ increases until $j^{\prime}\left(\lambda_{0}\right)=0$ for some finite number $\lambda_{0}>0$ and (2.50) holds on $\left[0, \lambda_{0}\right]$.

By $K_{2,-1}\left(\varphi_{2,-1}^{\lambda_{0}}\right)=j^{\prime}\left(\lambda_{0}\right)=0$, we know that

$$
E\left(\varphi_{2,-1}^{\lambda_{0}}\right) \geq m
$$

Now integrating (2.50) over $\left[0, \lambda_{0}\right]$, we obtain that

$$
-K_{2,-1}(\varphi)=j^{\prime}\left(\lambda_{0}\right)-j^{\prime}(0) \leq-\frac{8}{3}\left(j\left(\lambda_{0}\right)-j(0)\right) \leq-\frac{8}{3}(m-E(\varphi)),
$$

which implies (2.45). 
On the other hand, we have by (2.42)

$$
\frac{1}{4} \int_{\mathbb{R}^{4}}|\nabla \varphi|^{2} d x \leq E(\varphi)<m=\frac{1}{4} \int_{\mathbb{R}^{4}}|\nabla W|^{2} d x,
$$

and so (2.46) follows.

As a consequence of Lemma 2.13 energy conservation, continuous argument and Lemma 2.11, we deduce the following lemma.

Lemma 2.14. Let $(\alpha, \beta) \in \Omega$. Let $u: I \times \mathbb{R}^{4} \rightarrow \mathbb{C}$ be the solution to (CNLS) with initial data $u(0, x)=u_{0}(x) \in H^{1}\left(\mathbb{R}^{4}\right)$. Then, we have

(1) If $u_{0} \in \mathcal{K}_{\alpha, \beta}^{-}$, then for each $t \in I, u(t) \in \mathcal{K}_{\alpha, \beta}^{-}$and

$$
\|\nabla u(t)\|_{L_{x}^{2}}>\|\nabla W\|_{L_{x}^{2}} .
$$

(2) If $u_{0} \in \mathcal{K}_{\alpha, \beta}^{+}$, then for each $t \in I, u(t) \in \mathcal{K}_{\alpha, \beta}^{+}$and

$$
\|\nabla u(t)\|_{L_{x}^{2}}<\|\nabla W\|_{L_{x}^{2}} .
$$

Remark 2.15. By sharp Sobolev embedding: $\|\varphi\|_{L_{x}^{4}}^{4} \leq \frac{\|\nabla \varphi\|_{L_{x}^{2}}^{4}}{\|\nabla W\|_{L_{x}^{2}}^{4}}$, we know that if $\|\nabla \varphi\|_{L_{x}^{2}}<\|\nabla W\|_{L_{x}^{2}}$, then

$$
E_{c}(\varphi)=\frac{1}{2} \int_{\mathbb{R}^{4}}|\nabla \varphi|^{2} d x-\frac{1}{4} \int_{\mathbb{R}^{4}}|\varphi|^{4} d x>\frac{1}{4} \int_{\mathbb{R}^{4}}|\nabla \varphi|^{2}>0 .
$$

\section{BlOW UP OF $\mathcal{K}^{-}$}

In this section, we will prove that the radial solution of (CNLS) in $\mathcal{K}^{-}$blows up at finite time.

Let $\phi$ be a smooth, radial function satisfying $\left|\partial_{r}^{2} \phi(r)\right| \leq 2, \phi(r)=r^{2}$ for $r \leq 1$, and $\phi(r)=0$ for $r \geq 3$. For $R \geq 1$, we define

$$
\phi_{R}(x)=R^{2} \phi\left(\frac{|x|}{R}\right) \text { and } V_{R}(x)=\int_{\mathbb{R}^{4}} \phi_{R}(x)|u(t, x)|^{2} d x .
$$

Let $u(t, x)$ be a radial solution to (CNLS), then by a direct computation, we have

$$
\partial_{t} V_{R}(x)=2 \operatorname{Im} \int_{\mathbb{R}^{4}}\left[\bar{u} \partial_{j} u\right](t, x) \partial_{j} \phi_{R}(x) d x,
$$

and

$$
\begin{aligned}
\partial_{t}^{2} V_{R}(x)= & 4 \operatorname{Re} \int_{\mathbb{R}^{4}} \partial_{j} \partial_{k} \phi_{R}(x) \partial_{k} \bar{u}(t, x) \partial_{j} u(t, x) d x \\
& -\int_{\mathbb{R}^{4}}\left[\Delta^{2} \phi_{R}|u|^{2}+\Delta \phi_{R}\left(|u|^{4}-\frac{4}{5}|u|^{\frac{10}{3}}\right)\right] d x \\
= & 4 \int_{\mathbb{R}^{4}} \phi_{R}^{\prime \prime}|\nabla u|^{2} d x-\int_{\mathbb{R}^{4}}\left[\Delta^{2} \phi_{R}|u(t, x)|^{2}+\Delta \phi_{R}(x)\left(|u|^{4}-\frac{4}{5}|u|^{\frac{10}{3}}\right)(t, x)\right] d x \\
\leq & 4 \int_{\mathbb{R}^{4}}\left[2|\nabla u|^{2}-2|u|^{4}+\frac{8}{5}|u|^{\frac{10}{3}}\right](t, x) d x \\
& +\frac{C}{R^{2}} \int_{R \leq|x| \leq 3 R}|u|^{2} d x+C \int_{R \leq|x| \leq 3 R}\left[|u|^{4}+|u|^{\frac{10}{3}}\right] d x .
\end{aligned}
$$


By the radial Sobolev inequality, we have

$$
\|f\|_{L^{\infty}(|x| \geq R)} \leq \frac{c}{R^{\frac{3}{2}}}\|f\|_{L_{x}^{2}(|x| \geq R)}^{\frac{1}{2}}\|\nabla f\|_{L_{x}^{2}(|x| \geq R)}^{\frac{1}{2}} .
$$

Therefore, by mass conservation and Young's inequality, we know that for any $\epsilon>0$ there exist sufficiently large $R$ such that

$$
\begin{aligned}
\partial_{t}^{2} V(t) & \leq 8 K_{2,-1}(u(t))+\epsilon\|\nabla u(t, x)\|_{L^{2}}^{2}+\epsilon^{2} \\
& =32 E(u(t))-(8-\epsilon)\|\nabla u(t, x)\|_{L^{2}}^{2}-\frac{16}{5}\|u\|_{L_{x}^{\frac{10}{3}}}^{\frac{10}{3}}+\epsilon^{2} .
\end{aligned}
$$

This together with Lemma 2.14 and $E(u)<(1-\delta) m$ for some $\delta>0$ implies that

$$
\partial_{t}^{2} V_{R}(t) \leq-32 \delta m+C \varepsilon\|\nabla W\|_{L^{2}}^{2}+C \varepsilon^{2} .
$$

Finally, if we choose $\varepsilon$ sufficient small, we can obtain $\partial_{t}^{2} V_{R}(t) \leq-16 \delta_{2} m$, which implies that $u$ blows up in finite time.

\section{Profile DeCOMPosition}

In this section, we give the profile decomposition of (CNLS) by the strategy in [3, 16, 22, 23, 24. First, we give some notations for later use.

For $j, n \in \mathbb{N}$, we denote $T_{n}^{j}$ by $T_{n}^{j} \varphi(x)=\frac{1}{h_{n}^{j}} \varphi\left(\frac{x-x_{n}^{j}}{h_{n}^{j}}\right)$, where $\varphi \in H^{1}\left(\mathbb{R}^{4}\right)$ and $\left(x_{n}^{j}, h_{n}^{j}\right) \in \mathbb{R}^{4} \times(0,1]$. We define $\tau_{n}^{j}=\frac{t_{n}^{j}}{\left(h_{n}^{j}\right)^{2}}$ and the multiplier

$$
\sigma_{n}^{j}=\frac{\left\langle\left(h_{n}^{j}\right)^{-1} \nabla\right\rangle^{-1}\langle\nabla\rangle}{h_{n}^{j}} .
$$

4.1. Linear profile decomposition. Now we give the linear profile decomposition for the solutions sequence to free Schrödinger equation in the inhomogeneous space $H^{1}\left(\mathbb{R}^{4}\right)$.

Proposition 4.1 (Linear profile decomposition). Let $v_{n}(t, x)=e^{i t \Delta} \varphi_{n}(x)$ be a sequence of the free Schrödinger solutions with bounded $H^{1}\left(\mathbb{R}^{4}\right)$ norm. Then up to a subsequence, there exist $K \in\{1,2, \cdots, \infty\},\left\{\varphi^{j}\right\}_{j \in[1, K)} \subset H^{1}\left(\mathbb{R}^{4}\right)$ and $\left\{t_{n}^{j}, x_{n}^{j}, h_{n}^{j}\right\}_{n \in \mathbb{N}} \subset \mathbb{R} \times \mathbb{R}^{4} \times(0,1]$ satisfying

$$
v_{n}(t, x)=\sum_{j=1}^{k} v_{n}^{j}(t, x)+e^{i t \Delta} \omega_{n}^{k}, \text { for any } k \in[1, K),
$$

where

$$
\begin{aligned}
v_{n}^{j}(t, x) & =\langle\nabla\rangle^{-1}|\nabla| e^{i t \Delta} T_{n}^{j} e^{-i \tau_{n}^{j} \Delta}\langle\nabla\rangle|\nabla|^{-1} \varphi^{j} \\
& =e^{i t \Delta} T_{n}^{j} \sigma_{n}^{j} e^{-i \tau_{n}^{j} \Delta} \varphi^{j} \\
& =e^{i\left(t-t_{n}^{j}\right) \Delta} T_{n}^{j} \sigma_{n}^{j} \varphi^{j}
\end{aligned}
$$

and $\left\{\omega_{n}^{k}: k \in[1, K), n \geq 1\right\} \subset H^{1}\left(\mathbb{R}^{4}\right)$. The error terms $\omega_{n}^{k}$ of the linear profile decomposition (4.2) have the properties:

$$
\lim _{k \rightarrow K} \lim _{n \rightarrow \infty}\left\||\nabla|^{-1}\langle\nabla\rangle e^{i t \Delta} \omega_{n}^{k}\right\|_{L_{t}^{q}\left(\mathbb{R} ; L^{r}\left(\mathbb{R}^{4}\right)\right)}=0, \text { for any } \dot{H}^{1} \text {-admissible pair }(q, r),
$$


and for any $j \leq k \in[1, K)$.

$$
\lim _{n \rightarrow \infty} e^{i \tau_{n}^{j} \Delta}\left(T_{n}^{j}\right)^{-1}|\nabla|^{-1}\langle\nabla\rangle \omega_{n}^{k}=0 \quad \text { weakly in } \dot{H}^{1}\left(\mathbb{R}^{4}\right) .
$$

For any $l<j \leq K$, and $k \in[1, K)$, we have

$$
\begin{aligned}
& \lim _{n \rightarrow \infty}\left(\left|\log \frac{h_{n}^{l}}{h_{n}^{j}}\right|+\left|\frac{t_{n}^{j}-t_{n}^{l}}{\left(h_{n}^{l}\right)^{2}}\right|+\left|\frac{x_{n}^{j}-x_{n}^{l}}{h_{n}^{l}}\right|\right)=\infty, \\
& \lim _{n \rightarrow+\infty}\left|M\left(v_{n}(0)\right)-\sum_{j=1}^{k} M\left(v_{n}^{j}(0)\right)-M\left(\omega_{n}^{k}\right)\right|=0, \\
& \lim _{n \rightarrow+\infty}\left|E\left(v_{n}(0)\right)-\sum_{j=1}^{k} E\left(v_{n}^{j}(0)\right)-E\left(\omega_{n}^{k}\right)\right|=0,
\end{aligned}
$$

and

$$
\lim _{n \rightarrow+\infty}\left|K_{2,-1}\left(v_{n}(0)\right)-\sum_{j=1}^{k} K_{2,-1}\left(v_{n}^{j}(0)\right)-K_{2,-1}\left(\omega_{n}^{k}\right)\right|=0 .
$$

Moreover, for each fixed $j$, the sequence $\left\{h_{n}^{j}\right\}_{n \in N}$ is either going to 0 or identically 1 for all $n$.

Note that, by $k \in[1, K)$ we mean that $1 \leq k \leq K$ if $K<+\infty$ and $1 \leq k<\infty$ if $K=\infty$.

Proof. The proof is given by [3, Lemma 2.2] based on [22, Theroem 1.6], where the linear profile decomposition dealt with the sequences in $\dot{H}^{1}$. And the property (4.5) is from [20, lemma 2.10].

We also have the following property for a free Schrödinger solution sequence if it is in $\mathcal{K}^{+}$.

Corollary 4.2. Suppose that $v_{n}(t, x)$ is a sequence of the free Schrödinger solution with bounded $H^{1}\left(\mathbb{R}^{4}\right)$ norm satisfying that

$$
v_{n}(0) \in \mathcal{K}^{+} \text {and } E\left(v_{n}(0)\right) \leq m_{0}<m .
$$

Let

$$
v_{n}(t, x)=\sum_{j=1}^{k} v_{n}^{j}(t, x)+e^{i t \Delta} \omega_{n}^{k}
$$

be the linear profile decomposition given by Proposition 4.1. Then for sufficiently large $n$ and all $j, k \in[1, K)$, we have $v_{n}^{j}(0), w_{n}^{k}(x) \in \mathcal{K}^{+}$. Moreover for all $j<K$, we have

$$
0 \leq \varliminf_{n \rightarrow \infty} E\left(v_{n}^{j}(0)\right) \leq \varlimsup_{n \rightarrow \infty} E\left(v_{n}^{j}(0)\right) \leq \varliminf_{n \rightarrow \infty} E\left(v_{n}(0)\right),
$$

where the last inequality becomes equality only if $K=1$ and $\omega_{n}^{1}(x) \rightarrow 0$ in $\dot{H}^{1}$ as $n \rightarrow \infty$. 
Proof. Since $v_{n}(0) \in \mathcal{K}^{+}$and $E\left(v_{n}(0)\right) \leq m_{0}$, by Lemma 2.14, we have

$$
\varlimsup_{n \rightarrow \infty}\left\|\nabla v_{n}(0)\right\|_{L^{2}\left(\mathbb{R}^{4}\right)}<\|\nabla W\|_{L^{2}\left(\mathbb{R}^{4}\right)} .
$$

And from the linear profile decomposition, up to a subsequence, we have

$$
\lim _{n \rightarrow \infty}\left(\left\|v_{n}^{j}(0)\right\|_{H^{1}\left(\mathbb{R}^{4}\right)}^{2}+\left\|\omega_{n}^{k}\right\|_{H^{1}\left(\mathbb{R}^{4}\right)}^{2}\right) \leq \lim _{n \rightarrow \infty}\left\|v_{n}(0)\right\|_{H^{1}\left(\mathbb{R}^{4}\right)}^{2}<\infty,
$$

and $\left\|v_{n}^{j}(0)\right\|_{\dot{H}^{1}\left(\mathbb{R}^{4}\right)}^{2}+\left\|\omega_{n}^{k}\right\|_{\dot{H}^{1}\left(\mathbb{R}^{4}\right)}^{2}<\|W\|_{\dot{H}^{1}\left(\mathbb{R}^{4}\right)}^{2}$ for large $n$ and any $j<k \in[1, K)$.

Then also by Lemma 2.14 we have $v_{n}^{j}(0)$ and $\omega_{n}^{k}$ positive energy, which together with (4.8) implies (4.10). Thus, $v_{n}^{j}(0), w_{n}^{k} \in \mathcal{K}^{+}$follows from the definition of $\mathcal{K}^{+}$.

4.2. Nonlinear Profile decomposition. Now we are ready to give the construction of the nonlinear profile decomposition. The strategy here is the same as the $3 \mathrm{D}$ case in [23] and higher dimensional case in [3, 24].

Let $v_{n}(t, x)$ and $u_{n}(t, x)$ be solutions to the free Schrödinger equation and the (CNLS) respectively. And assume that $v_{n}(t, x)$ and $u_{n}(t, x)$ have the same initial datum $\varphi_{n}(x)$. By Proposition 4.1, we have the linear profile decomposition

$$
\begin{aligned}
v_{n}(t, x) & =\sum_{j=1}^{k} v_{n}^{j}(t, x)+e^{i t \Delta} \omega_{n}^{k} \\
& =\sum_{j=1}^{k} e^{i\left(t-t_{n}^{j}\right) \Delta} T_{n}^{j} \sigma_{n}^{j} \varphi^{j}+e^{i t \Delta} \omega_{n}^{k} .
\end{aligned}
$$

Let $u_{n}^{j}(t, x)$ be the solution to (CNLS) with the initial data $u_{n}^{j}(0, x)=v_{n}^{j}(0, x)$. If we define $U_{n}^{j}(t, x)$ by $u_{n}^{j}(t, x)=T_{n}^{j} \sigma_{n}^{j} U_{n}^{j}\left(\frac{t-t_{n}^{j}}{\left(h_{n}^{j}\right)^{2}}\right)$, then $U_{n}^{j}$ satisfies

$$
\left\{\begin{aligned}
\left(i \partial_{t}+\Delta\right) U_{n}^{j} & =-\left(\sigma_{n}^{j}\right)^{-1} f_{1}\left(\sigma_{n}^{j} U_{n}^{j}\right)+\left(h_{n}^{j}\right)^{\frac{2}{3}}\left(\sigma_{n}^{j}\right)^{-1} f_{2}\left(\sigma_{n}^{j} U_{n}^{j}\right) \\
U_{n}^{j}\left(-\tau_{n}^{j}\right) & =e^{-i \tau_{n}^{j} \Delta} \varphi^{j}
\end{aligned}\right.
$$

with $f_{1}(u)=|u|^{2} u$ and $f_{2}(u)=|u|^{\frac{10}{3}} u$.

Next, let $\left\{U_{\infty}^{j}\right\}_{j \geq 1}$ be the solutions of the limit equation of above equation. More precisely, for each $j$, we denote the limit of $\tau_{n}^{j}$ and $\sigma_{n}^{j}$ by

$$
\tau_{\infty}^{j}=\lim _{n \rightarrow \infty} \tau_{n}^{j} \in[-\infty,+\infty],
$$

and

$$
\sigma_{\infty}^{j}=\left\{\begin{array}{l}
1, \quad h_{n}^{j} \equiv 1 \\
\frac{\langle\nabla\rangle}{|\nabla|}, \quad h_{n}^{j} \rightarrow 0 \text { as } n \rightarrow \infty
\end{array}\right.
$$

Then $U_{\infty}^{j}$ satisfies

$$
\left\{\begin{aligned}
\left(i \partial_{t}+\Delta\right) U_{\infty}^{j} & =-f_{1}\left(U_{\infty}^{j}\right)+f_{2}\left(U_{\infty}^{j}\right) \\
U_{\infty}^{j}\left(-\tau_{\infty}^{j}\right) & =e^{-i \tau_{\infty}^{j} \Delta} \varphi^{j}
\end{aligned}\right.
$$

when $h_{n}^{j} \equiv 1$, or

$$
\left\{\begin{aligned}
\left(i \partial_{t}+\Delta\right) U_{\infty}^{j} & =-\left(\sigma_{\infty}^{j}\right)^{-1} f_{1}\left(\sigma_{\infty}^{j} U_{\infty}^{j}\right) \\
U_{\infty}^{j}\left(-\tau_{\infty}^{j}\right) & =e^{-i \tau_{\infty}^{j} \Delta} \varphi^{j},
\end{aligned}\right.
$$


when $h_{n}^{j} \rightarrow 0$ as $n \rightarrow \infty$. When $\tau_{\infty}^{j}= \pm \infty$, we consider (4.14) and (4.15) as the final value problems, which means that $U_{\infty}^{j}(t, x)$ satisfies

$$
\lim _{n \rightarrow \infty}\left\|U_{\infty}^{j}\left(-\tau_{n}^{j}\right)-e^{-i \tau_{n}^{j} \Delta} \varphi^{j}\right\|_{H^{1}\left(\mathbb{R}^{4}\right)}=0 .
$$

Based on this, we define the nonlinear profile by $u_{(n)}^{j}(t, x)=T_{n}^{j} \sigma_{n}^{j} U_{\infty}^{j}\left(\frac{t-t_{n}^{j}}{\left(h_{n}^{j}\right)^{2}}\right)$, which satisfies

$$
\left\{\begin{aligned}
\left(i \partial_{t}+\Delta\right) u_{(n)}^{j} & =-f_{1}\left(u_{(n)}^{j}\right)+f_{2}\left(u_{(n)}^{j}\right) \\
u_{(n)}^{j}\left(t_{n}^{j}-\tau_{\infty}^{j}\right) & =e^{-i \tau_{\infty}^{j} \Delta} \varphi^{j}
\end{aligned}\right.
$$

when $h_{n}^{j} \equiv 1$, or

$$
\left\{\begin{array}{cl}
\left(i \partial_{t}+\Delta\right) u_{(n)}^{j} & =-\frac{|\nabla|}{\langle\nabla\rangle} f_{1}\left(\frac{\langle\nabla\rangle}{|\nabla|} u_{(n)}^{j}\right) \\
u_{(n)}^{j}\left(t_{n}^{j}-\tau_{\infty}^{j}\left(h_{n}^{j}\right)^{2}\right) & =e^{-i \tau_{\infty}^{j} \Delta \varphi^{j},}
\end{array}\right.
$$

when $h_{n}^{j} \rightarrow 0$ as $n \rightarrow \infty$. Then we define the nonlinear profile decomposition of $u_{n}(t, x)$ by

$$
\tilde{u}_{n}^{k}:=\sum_{j=1}^{k} u_{(n)}^{j}+e^{i t \Delta} \omega_{n}^{k} .
$$

Next we will show that each nonlinear profile such that $u_{(n)}^{j} \in \mathcal{K}^{+}$, if $u_{n}(0) \in \mathcal{K}^{+}$ for each $n$.

Lemma 4.3. Suppose $u_{n}(t, x)$ is a solution sequence of the (CNLS) with the initial datum $u_{n}(0)$, which is bounded in $H^{1}\left(\mathbb{R}^{4}\right)$ with $u_{n}(0) \in \mathcal{K}^{+}$. Then we have the nonlinear decomposition given by

$$
\tilde{u}_{n}^{k}=\sum_{j=1}^{k} u_{(n)}^{j}+e^{i t \Delta} \omega_{n}^{k} .
$$

Let $I^{j}$ be the maximal lifespan of $U_{\infty}^{j}$, Then for $t \in I^{j}$, we have that

$$
\sigma_{\infty}^{j} U_{\infty}^{j}(t) \in \begin{cases}\mathcal{K}^{+}, & h_{n}^{j} \equiv 1 ; \\ \mathcal{K}_{c}^{+}, & h_{n}^{j} \rightarrow 0 \text { as } n \rightarrow \infty\end{cases}
$$

where $\mathcal{K}_{c}^{+}$is defined by

$$
\mathcal{K}_{c}^{+}:=\left\{\varphi \in \dot{H}^{1}\left(\mathbb{R}^{4}\right): E_{c}(\varphi)<E_{c}(W),\|\nabla \varphi\|_{L_{x}^{2}}^{2} \geq\|\varphi\|_{L_{x}^{4}}^{4}\right\} .
$$

Proof. By Corollary 4.2, we have that $v_{n}^{j}(0)=e^{-i t_{n}^{j} \Delta} T_{n}^{j} \sigma_{n}^{j} \varphi^{j} \in \mathcal{K}^{+}$. Then from the definition of $T_{n}^{j}$ and the fact that $\left|h_{n}^{j}\right| \leq 1$, we have by Lemma 2.14

$$
\left\|e^{-i \tau_{n}^{j} \Delta} \sigma_{n}^{j} \varphi^{j}\right\|_{\dot{H}^{1}\left(\mathbb{R}^{4}\right)}=\left\|e^{-i t_{n}^{j} \Delta} T_{n}^{j} \sigma_{n}^{j} \varphi^{j}\right\|_{\dot{H}^{1}\left(\mathbb{R}^{4}\right)}<\|W\|_{\dot{H}^{1}\left(\mathbb{R}^{4}\right)} .
$$

If $h_{n}^{j} \equiv 1$, then $\sigma_{n}^{j}=\sigma_{\infty}^{j} \equiv 1$. Hence from (4.16), we have $\sigma_{\infty}^{j} U_{\infty}^{j}\left(-\tau_{n}^{j}\right) \in \mathcal{K}^{+}$, for large $n$. By Lemma 2.14 we have $\sigma_{\infty}^{j} U_{\infty}^{j}(t) \in \mathcal{K}^{+}$for $t \in I^{j}$.

On the other hand, if $h_{n}^{j} \rightarrow 0$, then we have

$$
\left\|\sigma_{\infty}^{j} U_{\infty}^{j}\left(-\tau_{\infty}^{j}\right)\right\|_{\dot{H}^{1}\left(\mathbb{R}^{4}\right)}=\lim _{n \rightarrow \infty}\left\|\sigma_{\infty}^{j} e^{-i \tau_{n}^{j} \Delta} \varphi^{j}\right\|_{\dot{H}^{1}\left(\mathbb{R}^{4}\right)}<\|W\|_{\dot{H}^{1}\left(\mathbb{R}^{4}\right)} .
$$


This implies $E_{c}\left(\sigma_{\infty}^{j} U_{\infty}^{j}\left(-\tau_{\infty}^{j}\right)\right)>0$ by Remark 2.15. And by the Sobolev embedding $\dot{H}^{1}\left(\mathbb{R}^{4}\right) \hookrightarrow L^{4}\left(\mathbb{R}^{d}\right)$, we have

$$
E_{c}\left(\sigma_{\infty}^{j} U_{\infty}^{j}\left(-\tau_{\infty}^{j}\right)\right)=\lim _{n \rightarrow \infty} E_{c}\left(\sigma_{n}^{j} e^{-i \tau_{n}^{j} \Delta} \varphi^{j}\right)=\lim _{n \rightarrow \infty} E_{c}\left(v_{n}^{j}(0)\right)<E_{c}(W) .
$$

Hence, if $t \in I^{j}$, we have $\sigma_{\infty}^{j} U_{\infty}^{j}(t) \in \mathcal{K}_{c}^{+}$, which ends the proof.

Lemma 4.4. Let $U_{\infty}^{j}$ be constructed by above discussion and $I^{j}$ be the maximal lifespan of $U_{\infty}^{j}$. Then there exists $j_{0}$ large enough such that the following property:

$$
\sum_{j \geq j_{0}}\left\|\langle\nabla\rangle U_{\infty}^{j}\right\|_{S(\mathbb{R})}^{2} \leq \sum_{j \geq j_{0}}\left\|\varphi^{j}\right\|_{H^{1}\left(\mathbb{R}^{4}\right)}^{2}<\infty .
$$

Proof. For any $\delta>0$, by the linear decomposition, there exist two constants $j_{0}$ and $n_{0}$ large enough with the property that: If $n \geq n_{0}$, then we have

$$
\sum_{j \geq j_{0}}\left\|e^{-i \tau_{n}^{j} \Delta} \varphi^{j}\right\|_{H^{1}\left(\mathbb{R}^{4}\right)}^{2}=\sum_{j \geq j_{0}}\left\|\varphi^{j}\right\|_{H^{1}\left(\mathbb{R}^{4}\right)}^{2}=\sum_{j \geq j_{0}}\left\|T_{n}^{j} e^{-i \tau_{n}^{j} \Delta} \sigma_{n}^{j} \varphi^{j}\right\|_{H^{1}\left(\mathbb{R}^{4}\right)}^{2}<\delta .
$$

If $h_{n}^{j} \equiv 1$, by small data theory of (CNLS), we have

$$
\left\|\langle\nabla\rangle U_{\infty}^{j}\right\|_{S(\mathbb{R})}^{2} \lesssim\left\|\varphi^{j}\right\|_{H^{1}\left(\mathbb{R}^{4}\right)}^{2} .
$$

If $h_{n}^{j} \rightarrow 0$, by the Strichartz estimates and the Mikhlin multiplier theorem, we have

$$
\begin{aligned}
\left\|\langle\nabla\rangle U_{\infty}^{j}\right\|_{S(\mathbb{R})} & \lesssim\left\|\varphi^{j}\right\|_{H^{1}\left(\mathbb{R}^{4}\right)}+\left\||\nabla| f_{1}\left(\sigma_{\infty}^{j} U_{\infty}^{j}\right)\right\|_{L_{t}^{2}\left(\mathbb{R} ; L_{x}^{4 / 3}\left(\mathbb{R}^{4}\right)\right)} \\
& \lesssim\left\|\varphi^{j}\right\|_{H^{1}\left(\mathbb{R}^{4}\right)}+\left\|\sigma_{\infty}^{j} U_{\infty}^{j}\right\|_{W_{1}(R)}^{2}\left\|\langle\nabla\rangle U_{\infty}^{j}\right\|_{L_{t}^{6}\left(\mathbb{R} ; L_{x}^{12 / 5}\left(\mathbb{R}^{4}\right)\right)} \\
& \lesssim\left\|\varphi^{j}\right\|_{H^{1}\left(\mathbb{R}^{4}\right)}+\left\|\langle\nabla\rangle U_{\infty}^{j}\right\|_{S(\mathbb{R})}^{2}\left\|\langle\nabla\rangle U_{\infty}^{j}\right\|_{L_{t}^{2}\left(\mathbb{R} ; L_{x}^{4}\left(\mathbb{R}^{4}\right)\right)}^{1 / 3}\left\|\langle\nabla\rangle U_{\infty}^{j}\right\|_{L_{t}^{\infty}\left(\mathbb{R} ; L_{x}^{2}\left(\mathbb{R}^{4}\right)\right)}^{2 / 3} \\
& \lesssim\left\|\varphi^{j}\right\|_{H^{1}\left(\mathbb{R}^{4}\right)}+\left\|\langle\nabla\rangle U_{\infty}^{j}\right\|_{S(\mathbb{R})}^{3}
\end{aligned}
$$

Hence, by the continuity argument, for sufficiently small $\delta>0$, we have $\left\|\langle\nabla\rangle U_{\infty}^{j}\right\|_{S(\mathbb{R})} \lesssim$ $\left\|\varphi^{j}\right\|_{H^{1}\left(\mathbb{R}^{4}\right)}$. Combining this two cases together, we have

$$
\sum_{j \geq j_{0}}\left\|\langle\nabla\rangle U_{\infty}^{j}\right\|_{S(\mathbb{R})}^{2} \lesssim \sum_{j \geq j_{0}}\left\|\varphi^{j}\right\|_{H^{1}\left(\mathbb{R}^{4}\right)}^{2}
$$

This ends the proof of this lemma.

Recall $S T(I)=W_{1}(I) \cap W_{2}(I)$ in Section 2.1. Now we define the scattering size $\left\|\sigma_{\infty}^{j} U_{\infty}^{j}\right\|_{S T^{j}(I)}$ of $\sigma_{\infty}^{j} U_{\infty}^{j}$ for each $j$ by

$$
S T^{j}(I)=\left\{\begin{array}{lc}
W_{1}(I) \cap W_{2}(I), & \text { for } h_{n}^{j} \equiv 1 \\
W_{1}(I), & h_{\infty}^{j}=0 .
\end{array}\right.
$$

Lemma 4.5. Let $k_{0} \in \mathbb{N}$ such that for any $1 \leq j \leq k_{0}$,

$$
\left\|\sigma_{\infty}^{j} U_{\infty}^{j}\right\|_{S T^{j}\left(I^{j}\right)}<\infty
$$

Then we have $I^{j}=\infty$ and

$$
\left\|\nabla u_{(n)}^{j}\right\|_{V_{0}(\mathbb{R})}+\left\|u_{(n)}^{j}\right\|_{S T(\mathbb{R})} \lesssim\left\|\langle\nabla\rangle U_{\infty}^{j}\right\|_{S(\mathbb{R})} \lesssim 1, \text { for } 1 \leq j \leq k_{0} .
$$


And there exists $B>0$ such that: for any given $k \in \mathbb{N}$ and $1 \leq k \leq k_{0}$, there exists $N_{k} \in \mathbb{N}$ s.t

$$
\sup _{n \geq N_{k}}\left(\left\|\sum_{j=1}^{k} \nabla u_{(n)}^{j}\right\|_{V_{0}(\mathbb{R})}+\left\|\sum_{j=1}^{k} u_{(n)}^{j}\right\|_{S T(\mathbb{R})}\right) \leq B .
$$

Furthermore, if (4.24) holds for any $j \in \mathbb{N}$, then, for any $p \in\{12 / 7,2\}$ and $j \geq 1$, we have

$$
\lim _{k \rightarrow \infty} \lim _{n \rightarrow \infty}\left\|u_{(n)}^{j}|\nabla|^{s} e^{i t \Delta} \omega_{n}^{k}\right\|_{L_{t, x}^{p}\left(\mathbb{R}^{\times} \mathbb{R}^{4}\right)}=0, \text { and } s \in\{0,1\} .
$$

Proof. First, $I^{j}=\mathbb{R}$ follows from the local well-posedness theory of (4.14) and (4.15) and the scattering size condition of (4.24).

Next, for the first inequality in (4.25), by employing the Mikhlin multiplier theorem, we have

$$
\begin{aligned}
& \left\|u_{(n)}^{j}\right\|_{W_{1}(\mathbb{R})}=\left\|\sigma_{n}^{j} U_{\infty}^{j}\right\|_{W_{1}(\mathbb{R})} \lesssim\left\|\sigma_{\infty}^{j} U_{\infty}^{j}\right\|_{W_{1}(\mathbb{R})} \lesssim\left\|\langle\nabla\rangle U_{\infty}^{j}\right\|_{S(\mathbb{R})}, \\
& \left\|u_{(n)}^{j}\right\|_{W_{2}(\mathbb{R})} \\
& \quad \leq\left\|u_{(n)}^{j}\right\|_{W_{1}(\mathbb{R})}^{1 / 2}\left\|u_{(n)}^{j}\right\|_{V_{0}(\mathbb{R})}^{1 / 2} \\
& \\
& \quad \lesssim\left\|\langle\nabla\rangle U_{\infty}^{j}\right\|_{S(\mathbb{R})}^{1 / 2}\left(h_{n}^{j}\right)^{1 / 2}\left\|\sigma_{n}^{j} U_{\infty}^{j}\right\|_{V_{0}(\mathbb{R})}^{1 / 2} \\
& \quad \lesssim\left\|\langle\nabla\rangle U_{\infty}^{j}\right\|_{S(\mathbb{R})}, \\
& \text { and } \\
& \left\|\nabla u_{(n)}^{j}\right\|_{V_{0}(\mathbb{R})}=\left\|\nabla \sigma_{n}^{j} U_{\infty}^{j}\right\|_{V_{0}(\mathbb{R})} \lesssim\left\|\nabla \sigma_{\infty}^{j} U_{\infty}^{j}\right\|_{S(\mathbb{R})} \lesssim\left\|\langle\nabla\rangle U_{\infty}^{j}\right\|_{S(\mathbb{R})} .
\end{aligned}
$$

Hence to finish (4.25), it suffices to show

$$
\left\|\langle\nabla\rangle U_{\infty}^{j}\right\|_{S(\mathbb{R})} \lesssim 1
$$

If $h_{n}^{j} \rightarrow 0$, by using the Strichartz estimates, (4.16), and (4.24), we have

$$
\begin{aligned}
\left\|\langle\nabla\rangle U_{\infty}^{j}\right\|_{S(\mathbb{R})} & \lesssim\left\|\varphi^{j}\right\|_{H^{1}\left(\mathbb{R}^{4}\right)}+\left\|\langle\nabla\rangle\left(\sigma_{\infty}^{j}\right)^{-1} f_{1}\left(\sigma_{\infty}^{j} U_{\infty}^{j}\right)\right\|_{L_{t}^{2}\left(\mathbb{R} ; L_{x}^{4 / 3}\left(\mathbb{R}^{4}\right)\right)} \\
& \lesssim\left\|\varphi^{j}\right\|_{H^{1}\left(\mathbb{R}^{4}\right)}+\left\|\sigma_{\infty}^{j} U_{\infty}^{j}\right\|_{W_{1}(R)}^{2}\left\|\langle\nabla\rangle U_{\infty}^{j}\right\|_{L_{t}^{6}\left(\mathbb{R} ; L_{x}^{12 / 5}\left(\mathbb{R}^{4}\right)\right)} \\
& \lesssim\left\|\varphi^{j}\right\|_{H^{1}\left(\mathbb{R}^{4}\right)}+\left\|\langle\nabla\rangle U_{\infty}^{j}\right\|_{L_{t}^{2}\left(\mathbb{R} ; L_{x}^{4}\left(\mathbb{R}^{4}\right)\right)}^{1 / 3}\left\|\langle\nabla\rangle U_{\infty}^{j}\right\|_{L_{t}^{\infty}\left(\mathbb{R} ; L_{x}^{2}\left(\mathbb{R}^{4}\right)\right)}^{2 / 3}
\end{aligned}
$$

In this case, similar to Lemma 4.4. $\left\|\varphi^{j}\right\|_{H^{1}\left(\mathbb{R}^{4}\right)} \lesssim 1$ and by Lemma 4.3, we have $\left\|\langle\nabla\rangle U_{\infty}^{j}\right\|_{L_{t}^{\infty}\left(\mathbb{R} ; L_{x}^{2}\left(\mathbb{R}^{4}\right)\right)} \lesssim 1$. Thus, by the weighted Hödelr inequality, we have

$$
\left\|\langle\nabla\rangle \sigma_{\infty}^{j} U_{\infty}^{j}\right\|_{S(\mathbb{R})} \lesssim 1
$$

On the other hand, if $h_{n}^{j} \equiv 1$, then $\sigma_{\infty}^{j}=1$. Similar to the former case, by the condition (4.24), we have

$$
\begin{aligned}
\left\|\langle\nabla\rangle U_{\infty}^{j}\right\|_{S(\mathbb{R})} & \lesssim 1+\left\|\langle\nabla\rangle\left[f_{1}\left(U_{\infty}^{j}\right)+f_{2}\left(U_{\infty}^{j}\right)\right]\right\|_{L_{t}^{2}\left(\mathbb{R} ; L_{x}^{4 / 3}\left(\mathbb{R}^{4}\right)\right)} \\
& \lesssim 1 .
\end{aligned}
$$

By using the standard continuity argument again, we have (4.31) in this case, which implies (4.25). 
Second, to prove 4.26), we use

$$
\left.|| \sum_{1 \leq j \leq k} u_{(n)}^{j}\right|^{q}-\left.\sum_{1 \leq j \leq k}\left|u_{(n)}^{j}\right|^{q}\left|\lesssim_{k, q} \sum_{1 \leq j \leq k} \sum_{\substack{1 \leq j^{\prime} \leq k \\ j^{\prime} \neq j}}\right| u_{(n)}^{j}\right|^{q-1}\left|u_{(n)}^{j^{\prime}}\right|, \quad 1<q<\infty .
$$

to obtain

$$
\left\|\sum_{1 \leq j \leq k} u_{(n)}^{j}\right\|_{W_{1}(\mathbb{R})}^{6} \leq \sum_{1 \leq j \leq k}\left\|u_{(n)}^{j}\right\|_{W_{1}(\mathbb{R})}^{6}+C_{k} \sum_{1 \leq j \leq k} \sum_{\substack{1 \leq j^{\prime} \leq k \\ j^{\prime} \neq j}} \int_{\mathbb{R}} \int_{\mathbb{R}^{4}}\left|u_{(n)}^{j}\right|^{5}\left|u_{(n)}^{j^{\prime}}\right| d x d t .
$$

Without loss of generality, we assume that $k \geq j_{0}$ in Lemma 4.4 Hence, we have

$$
\begin{aligned}
\sum_{1 \leq j \leq k}\left\|u_{(n)}^{j}\right\|_{W_{1}(\mathbb{R})}^{6} & \lesssim \sum_{1 \leq j \leq j_{0}}\left\|u_{(n)}^{j}\right\|_{W_{1}(\mathbb{R})}^{6}+\sum_{j_{0}<j \leq k}\left\|u_{(n)}^{j}\right\|_{W_{1}(\mathbb{R})}^{6} \\
& \lesssim \sum_{1 \leq j \leq j_{0}}\left\|\langle\nabla\rangle U_{\infty}^{j}\right\|_{S(\mathbb{R})}^{6}+\sum_{j_{0}<j \leq k}\left\|\varphi^{j}\right\|_{H^{1}}^{2}<+\infty .
\end{aligned}
$$

On the other hand, by the almost decoupling condition (4.6), there exists $N_{k} \in \mathbb{N}$ such that

$$
\int_{\mathbb{R}} \int_{\mathbb{R}^{4}}\left|u_{(n)}^{j}\right|^{5}\left|u_{(n)}^{j^{\prime}}\right| d x d t \leq \frac{1}{C_{k} k^{2}}, \text { for } j \neq j^{\prime} \text { and } n \geq N_{k} .
$$

Hence, from (4.34), (4.35) and (4.36), there exists $B_{0}>0$, which suffices that for any $1 \leq k \leq k_{0}, \exists N_{k}^{\prime} \in N$ such that

$$
\sup _{n \geq N_{k}^{\prime}}\left\|\sum_{j=1}^{k} u_{(n)}^{j}\right\|_{W_{1}(\mathbb{R})} \leq B_{0} .
$$

Similarly, by the above elementary inequality, Lemma 4.4 and the almost decoupling condition (4.6), we can prove that there exists $B_{1}>0$, which guarantees that for any $1 \leq k \leq k_{0}, \exists N_{k}^{\prime \prime} \in N$ such that

$$
\sup _{n \geq N_{k}^{\prime \prime}}\left\|\sum_{j=1}^{k} \nabla u_{(n)}^{j}\right\|_{V_{0}(\mathbb{R})}+\sup _{n \geq N_{k}}\left\|\sum_{j=1}^{k} u_{(n)}^{j}\right\|_{W_{2}(\mathbb{R})} \leq B_{1}
$$

Hence, by taking $B=\max \left\{B_{0}, B_{1}\right\}$ and $N_{k}=\max \left\{N_{k}^{\prime}, N_{k}^{\prime \prime}\right\}$, we have (4.26).

Finally, we turn to the proof of (4.27). For the case $s=0$, using the Hölder inequality and the Mikhlin multiplier theorem, we have as $n \rightarrow \infty$

$$
\begin{aligned}
& \left\|u_{(n)}^{j} e^{i t \Delta} \omega_{n}^{k}\right\|_{L_{t, x}^{12 / 7} \cap L_{t, x}^{2}} \\
\lesssim & \left\|u_{(n)}^{j}\right\|_{V_{0}(\mathbb{R})}\left[\left\|e^{i t \Delta} \omega_{n}^{k}\right\|_{W_{1}(\mathbb{R})}+\left\|e^{i t \Delta} \omega_{n}^{k}\right\|_{W_{2}(\mathbb{R})}\right] \\
\lesssim & \left\|h_{n}^{j} \sigma_{n}^{j} U_{\infty}^{j}\right\|_{L_{t, x}^{3}}\left[\left\|e^{i t \Delta} \omega_{n}^{k}\right\|_{W_{1}(\mathbb{R})}+\left\|e^{i t \Delta} \omega_{n}^{k}\right\|_{W_{1}(\mathbb{R})}^{1 / 2}\left\|e^{i t \Delta} \omega_{n}^{k}\right\|_{V_{0}(\mathbb{R})}^{1 / 2}\right] \\
\lesssim & \left\|\langle\nabla\rangle U_{\infty}^{j}\right\|_{S(\mathbb{R})}\left\|e^{i t \Delta} \omega_{n}^{k}\right\|_{W_{1}(\mathbb{R})}^{1 / 2}\left[\left\|e^{i t \Delta} \omega_{n}^{k}\right\|_{W_{1}(\mathbb{R})}^{1 / 2}+\left\|\omega_{n}^{k}\right\|_{H^{1}\left(\mathbb{R}^{4}\right)}^{1 / 2}\right] \rightarrow 0,
\end{aligned}
$$

where we used the fact that (4.4) and $\omega_{n}^{k} \in H^{1}\left(\mathbb{R}^{4}\right)$ in Proposition 4.1. 
For the case $s=1$, from the fact that

$\left\|\sigma_{n}^{j} U_{\infty}^{j}-\sigma_{\infty}^{j} U_{\infty}^{j}\right\|_{W_{1}(\mathbb{R})} \lesssim \begin{cases}\left\|\left(\frac{h_{n}^{j}}{h_{n}^{j}+|\nabla|}\right)\langle\nabla\rangle U_{\infty}^{j}\right\|_{S(\mathbb{R})} \rightarrow 0, & \text { as } n \rightarrow \infty, \text { if } h_{n}^{j} \rightarrow 0 \\ 0, & h_{n}^{j} \equiv 1,\end{cases}$ we have that for any $\varepsilon>0$, we can find $v^{j} \in C_{c}^{\infty}\left(\mathbb{R} \times \mathbb{R}^{4}\right)$ and $N_{j} \in \mathbb{N}$ such that

$$
\left\|\sigma_{n}^{j} U_{\infty}^{j}-v^{j}\right\|_{W_{1}(\mathbb{R})} \leq \varepsilon \quad \text { for } \quad n>N_{j} .
$$

Hence, by the Hölder inequality and the Strichartz estimates we have

$$
\begin{aligned}
& \left\|u_{(n)}^{j}|\nabla| e^{i t \Delta} \omega_{n}^{k}\right\|_{L_{t, x}^{2}\left(\mathbb{R} \times \mathbb{R}^{4}\right)} \\
\lesssim & \left\|\sigma_{n}^{j} U_{\infty}^{j}\left[|\nabla| e^{i \cdot \Delta} \omega_{n}^{k}\right]\left(t\left(h_{n}^{j}\right)^{2}+t_{n}^{j}, x h_{n}^{j}+x_{n}^{j}\right)\right\|_{L_{t, x}^{2}\left(\mathbb{R} \times \mathbb{R}^{4}\right)}\left(h_{n}^{j}\right)^{2} \\
\lesssim & \left\|\sigma_{n}^{j} U_{\infty}^{j}-v^{j}\right\|_{W_{1}(\mathbb{R})}\left\|\omega_{n}^{k}\right\|_{H^{1}\left(\mathbb{R}^{4}\right)} \\
& +\left\|v^{j}\left[|\nabla| e^{i \cdot \Delta} \omega_{n}^{k}\right]\left(t\left(h_{n}^{j}\right)^{2}+t_{n}^{j}, x h_{n}^{j}+x_{n}^{j}\right)\right\|_{L_{t, x}^{2}\left(\mathbb{R} \times \mathbb{R}^{4}\right)}\left(h_{n}^{j}\right)^{2} .
\end{aligned}
$$

Assume supp $v^{j} \subset\left\{(t, x) \in \mathbb{R} \times \mathbb{R}^{4}:|t| \leq T_{j},|x| \leq R_{j}\right\}$ and let $\tilde{\omega}_{n}^{j, k}(x)=$ $e^{i \tau_{n}^{j} \Delta} \omega_{n}^{k}\left(x h_{n}^{j}+x_{n}^{j}\right)$, then by using Lemma 2.5 in [20] and [4.4) in Proposition 4.1] we have

$$
\begin{aligned}
& \left\|\left[|\nabla| e^{i\left(t\left(h_{n}^{j}\right)^{2}+t_{n}^{j}\right) \Delta} \omega_{n}^{k}\right]\left(x h_{n}^{j}+x_{n}^{j}\right)\right\|_{L_{t, x}^{2}\left(|t| \leq T_{j},|x| \leq R_{j}\right)}\left(h_{n}^{j}\right)^{2} \\
= & h_{n}^{j}\left\||\nabla| e^{i t \Delta} \tilde{\omega}_{n}^{j, k}\right\|_{L_{t, x}^{2}\left(|t| \leq T_{j},|x| \leq R_{j}\right)} \\
\lesssim & h_{n}^{j}\left(T_{j}\right)^{1 / 9}\left(R_{j}\right)^{1 / 18}\left\|e^{i t \Delta} \tilde{\omega}_{n}^{j, k}\right\|_{W_{1}(\mathbb{R})}^{1 / 3}\left\|\nabla \tilde{\omega}_{n}^{j, k}\right\|_{L_{x}^{2}\left(\mathbb{R}^{4}\right)}^{2 / 3} \\
\lesssim & \left(T_{j}\right)^{1 / 9}\left(R_{j}\right)^{1 / 18}\left\|e^{i t \Delta} \omega_{n}^{k}\right\|_{W_{1}(\mathbb{R})}^{1 / 3}\left\|\nabla \omega_{n}^{k}\right\|_{L_{x}^{2}\left(\mathbb{R}^{4}\right)}^{2 / 3} \rightarrow 0,
\end{aligned}
$$

as $k, n \rightarrow \infty$.

On the other hand, for $p=12 / 7$, we define

$$
V^{j}= \begin{cases}0, & h_{n}^{j} \rightarrow 0 \\ U_{\infty}^{j}, & h_{n}^{j} \equiv 1\end{cases}
$$

Then we have

$$
\left\|\left(h_{n}^{j}\right)^{1 / 2} \sigma_{n}^{j} U_{\infty}^{j}-V^{j}\right\|_{W_{2}(\mathbb{R})} \lesssim\left\||\nabla|^{1 / 2}\left(h_{n}^{j}\right)^{1 / 2} \sigma_{n}^{j} U_{\infty}^{j}-|\nabla|^{1 / 2} V^{j}\right\|_{V_{2}} \rightarrow 0,
$$

as $n \rightarrow \infty$. From this we can find $v^{j} \in C_{c}^{\infty}\left(\mathbb{R} \times \mathbb{R}^{4}\right)$ with the property that for any $\eta>0$, there exists $N_{j} \in \mathbb{N}$, such that $\left\|\left(h_{n}^{j}\right)^{1 / 2} \sigma_{n}^{j} U_{\infty}^{j}-v^{j}\right\|_{W_{2}(\mathbb{R})}<\eta$, if $n>N_{j}$.

By the Hölder inequality and the Strichartz estimates, we have

$$
\begin{aligned}
& \left\|u_{(n)}^{j}|\nabla| e^{i t \Delta} \omega_{n}^{k}\right\|_{L_{t, x}^{12 / 7}} \\
\lesssim & \left\|\left(h_{n}^{j}\right)^{1 / 2} \sigma_{n}^{j} U_{\infty}^{j} \cdot|\nabla| e^{i\left(t\left(h_{n}^{j}\right)^{2}+h_{n}^{j}\right) \Delta} \omega_{n}^{k}\left(x h_{n}^{j}+x_{n}^{j}\right)\right\|_{L_{t, x}^{12 / 7}}\left(h_{n}^{j}\right)^{2} \\
\lesssim & \left\||\nabla| e^{i\left(t\left(h_{n}^{j}\right)^{2}+h_{n}^{j}\right) \Delta} \omega_{n}^{k}\left(x h_{n}^{j}+x_{n}^{j}\right)\right\|_{L_{t}^{3}, x}\left(h_{n}^{j}\right)^{2} \\
& +\left\|v^{j}\left[|\nabla| e^{i t\left(h_{n}^{j}\right)^{2}+t_{n}^{j} \Delta} \omega_{n}^{k}\right]\left(x h_{n}^{j}+x_{n}^{j}\right)\right\|_{L_{t, x}^{12 / 7}\left(\mathbb{R} \times \mathbb{R}^{4}\right)}\left(h_{n}^{j}\right)^{2} \\
\lesssim & \left\||\nabla| \omega_{n}^{k}\right\|_{L^{2}\left(\mathbb{R}^{4}\right)}+\left\|v^{j}\left[|\nabla| e^{i t\left(h_{n}^{j}\right)^{2}+t_{n}^{j} \Delta} \omega_{n}^{k}\right]\left(x h_{n}^{j}+x_{n}^{j}\right)\right\|_{L_{t, x}^{2}\left(\mathbb{R} \times \mathbb{R}^{4}\right)}\left(h_{n}^{j}\right)^{2} .
\end{aligned}
$$


Similar to (4.41), we have the last term of (4.42) tends to 0 , as $k, n \rightarrow \infty$, which together with (4.40), (4.41) implies (4.27). This ends the proof of Lemma 4.5.

Proposition 4.6. Let $u_{n}^{j}$ and $U_{\infty}^{j}$ be as above. Assume that

$$
\left\|\sigma_{\infty}^{j} U_{\infty}^{j}\right\|_{S T^{j}\left(I^{j}\right)}<\infty, \quad \text { holds for any } j \geq 1 \text {. }
$$

Then we have

$$
\left\|u_{n}\right\|_{S T(\mathbb{R})}<\infty, \text { for sufficient large } n .
$$

Proof. To prove (4.44), we will use the perturbation theory (Proposition 2.5). More precisely, we need to prove that the nonlinear profile $\tilde{u}_{n}^{k}$ constructed above is actually a sequence of approximation solutions.

First, by Lemma 4.5 and our assumption of $U_{\infty}^{j}$ in (4.43), we have that $u_{(n)}^{j}$ is a global solution and $\tilde{u}_{n}^{k}$ is a function of $t$ defined on the whole $\mathbb{R}$.

Next, from the inequalities (4.26) in Lemma 4.5 and $H^{1}\left(\mathbb{R}^{4}\right)$ bounded condition of $\omega_{n}^{k}(x)$ in Corollary 4.2, we have that there exists a constant $B>0$ satisfying the property: there exists $N_{1, k}>0$, such that

$$
\sup _{n \geq N_{1, k}}\left\|\tilde{u}_{n}^{k}\right\|_{S T(\mathbb{R})} \leq B .
$$

In fact, the linear profile decomposition implies

$$
\begin{aligned}
\left\|u_{n}(0)-\tilde{u}_{n}^{k}(0)\right\|_{H^{1}\left(\mathbb{R}^{4}\right)} & \leq \sum_{j=1}^{k}\left\|v_{n}^{j}(0)-u_{(n)}^{j}(0)\right\|_{H^{1}\left(\mathbb{R}^{4}\right)} \\
& \lesssim \sum_{j=1}^{k}\left\|e^{-\tau_{n}^{j} \Delta} \varphi^{j}-U_{\infty}^{j}\left(-\tau_{n}^{j}\right)\right\|_{H^{1}\left(\mathbb{R}^{4}\right)} .
\end{aligned}
$$

From this and (4.16), we have that for any $k \in \mathbb{N}$, there exists $N_{2, k}>0$ such that

$$
\sup _{n \geq N_{2, k}}\left\|u_{n}(0)-\tilde{u}_{n}^{k}(0)\right\|_{H^{1}\left(\mathbb{R}^{4}\right)} \leq 1 .
$$

Similarly, by the Strichartz estimates and (4.16), for any $k \in \mathbb{N}$, we can find $N_{3, k} \in$ $\mathbb{N}$ such that

$$
\sup _{n \geq N_{3, k}}\left\|\langle\nabla\rangle e^{i t \Delta}\left(u_{n}(0)-\tilde{u}_{n}^{k}(0)\right)\right\|_{V_{2}(\mathbb{R})} \leq \delta,
$$

where the $\delta>0$ is from the perturbation theory and depends on $B$ and the uniform bound of $\left\|u_{n}(0)\right\|_{H^{1}\left(\left(\mathbb{R}^{4}\right)\right)}$.

Hence, by Proposition 2.5 (4.44) holds if we could show that there exist $k_{0}, N_{0} \in$ $\mathbb{N}$ such that if $k \geq k_{0}$ and $n \geq N_{0}$,

$$
\left\|\langle\nabla\rangle\left[\left(i \partial_{t}+\Delta\right) \tilde{u}_{n}^{k}+f_{1}\left(\tilde{u}_{n}^{k}\right)-f_{2}\left(\tilde{u}_{n}^{k}\right)\right]\right\|_{L_{t, x}^{\frac{3}{2}}\left(\mathbb{R} \times \mathbb{R}^{4}\right)} \leq \delta .
$$

To do this, if we define $f(u)=f_{1}(u)-f_{2}(u)$, then we have

$$
\begin{aligned}
\left(i \partial_{t}+\Delta\right) \tilde{u}_{n}^{k}+f\left(\tilde{u}_{n}^{k}\right)= & f\left(\tilde{u}_{n}^{k}\right)-f\left(\sum_{j=1}^{k} u_{(n)}^{j}\right) \\
& +\sum_{j=1}^{k}\left(i \partial_{t}+\Delta\right) u_{(n)}^{j}+f\left(\sum_{j=1}^{k} u_{(n)}^{j}\right) .
\end{aligned}
$$


Hence, we just need to show that

$$
\lim _{k \rightarrow \infty} \lim _{n \rightarrow \infty}\left\|\langle\nabla\rangle\left[f\left(\tilde{u}_{n}^{k}\right)-f\left(\sum_{j=1}^{k} u_{(n)}^{j}\right)\right]\right\|_{L_{t, x}^{\frac{3}{2}}\left(\mathbb{R} \times \mathbb{R}^{4}\right)}=0,
$$

and

$$
\lim _{k \rightarrow \infty} \lim _{n \rightarrow \infty}\left\|\langle\nabla\rangle\left[\sum_{j=1}^{k}\left(i \partial_{t}+\Delta\right) u_{(n)}^{j}+f\left(\sum_{j=1}^{k} u_{(n)}^{j}\right)\right]\right\|_{L_{t, x}^{\frac{3}{2}}\left(\mathbb{R} \times \mathbb{R}^{4}\right)}=0 .
$$

First, we will prove (4.51). Let $u(x)$ and $w(x)$ be two functions defined on $\mathbb{R}^{4}$, then if $1<r \leq 2$, we have

$$
|| u+\left.w\right|^{r}(u+w)-|u|^{r} u\left|\lesssim\left(|u|^{r}+|w|^{r}\right)\right| w \mid,
$$

and

$$
\left|\nabla\left(|u+w|^{r}(u+w)-|u|^{r} u\right)\right| \lesssim|u|^{r}|\nabla w|+(|\nabla u|+|\nabla w|)|w|^{r} .
$$

By these two inequalities and the Hölder inequality, to prove (4.51), we just need to estimate

$$
\begin{gathered}
\\
\left\|\left[\left|e^{i t \Delta} \omega_{n}^{k}\right|^{\frac{4}{3}}+\left|e^{i t \Delta} \omega_{n}^{k}\right|^{2}\right]\left|e^{i t \Delta} \omega_{n}^{k}\right|\right\|_{L_{t, x}^{\frac{3}{2}}\left(\mathbb{R} \times \mathbb{R}^{4}\right)} \\
+\left\|\left[\left|e^{i t \Delta} \omega_{n}^{k}\right|^{\frac{4}{3}}+\left|e^{i t \Delta} \omega_{n}^{k}\right|^{2}\right]\left|\nabla e^{i t \Delta} \omega_{n}^{k}\right|\right\|_{L_{t, x}^{\frac{3}{2}}\left(\mathbb{R} \times \mathbb{R}^{4}\right)}, \\
\left\|\left[\left|e^{i t \Delta} \omega_{n}^{k}\right|^{\frac{4}{3}}+\left|e^{i t \Delta} \omega_{n}^{k}\right|^{2}\right]\left|\sum_{j=1}^{k} \nabla u_{(n)}^{j}\right| \mid\right\|_{L_{t, x}^{\frac{3}{2}\left(\mathbb{R} \times \mathbb{R}^{4}\right)}},
\end{gathered}
$$

and

$$
\begin{gathered}
\left\|\left[\left|\sum_{j=1}^{k} u_{(n)}^{j}\right|^{\frac{4}{3}}+\left|\sum_{j=1}^{k} u_{(n)}^{j}\right|^{2}\right]\left|e^{i t \Delta} \omega_{n}^{k}\right|\right\|_{L_{t, x}^{\frac{3}{2}}\left(\mathbb{R} \times \mathbb{R}^{4}\right)} \\
+\left\|\left[\left|\sum_{j=1}^{k} u_{(n)}^{j}\right|^{\mid \frac{4}{3}}+\left|\sum_{j=1}^{k} u_{(n)}^{j}\right|^{2}\right]\left|\nabla e^{i t \Delta} \omega_{n}^{k}\right|\right\|_{L_{t, x}^{\frac{3}{2}}\left(\mathbb{R} \times \mathbb{R}^{4}\right)} .
\end{gathered}
$$

Now deal with (4.55). Using the Hölder inequalities, we have

$$
\begin{gathered}
\left\|\left|e^{i t \Delta} \omega_{n}^{k}\right|^{4 / 3}\left|e^{i t \Delta} \omega_{n}^{k}\right|\right\|_{L_{t, x}^{\frac{3}{2}}\left(\mathbb{R} \times \mathbb{R}^{4}\right)} \leq\left\|e^{i t \Delta} \omega_{n}^{k}\right\|_{W_{1}(\mathbb{R})}^{2 / 3}\left\|e^{i t \Delta} \omega_{n}^{k}\right\|_{V_{0}(\mathbb{R})}^{5 / 3}, \\
\left\|\left|e^{i t \Delta} \omega_{n}^{k}\right|^{2}\left|e^{i t \Delta} \omega_{n}^{k}\right|\right\|_{L_{t, x}^{\frac{3}{2}}\left(\mathbb{R} \times \mathbb{R}^{4}\right)} \leq\left\|e^{i t \Delta} \omega_{n}^{k}\right\|_{W_{1}(\mathbb{R})}^{2}\left\|e^{i t \Delta} \omega_{n}^{k}\right\|_{V_{0}(\mathbb{R})}, \\
\left\|\left.|| e^{i t \Delta} \omega_{n}^{k}\right|^{4 / 3} \mid \nabla e^{i t \Delta} \omega_{n}^{k}\right\|_{L_{t, x}^{\frac{3}{2}}\left(\mathbb{R} \times \mathbb{R}^{4}\right)} \\
\leq\left\|e^{i t \Delta} \omega_{n}^{k}\right\|_{W_{1}(\mathbb{R})}^{2 / 3}\left\|e^{i t \Delta} \omega_{n}^{k}\right\|_{V_{0}(\mathbb{R})}^{2 / 3}\left\|\nabla e^{i t \Delta} \omega_{n}^{k}\right\|_{V_{0}(\mathbb{R})},
\end{gathered}
$$

and

$$
\left\|\left|e^{i t \Delta} \omega_{n}^{k}\right|^{2}\left|\nabla e^{i t \Delta} \omega_{n}^{k}\right|\right\|_{L_{t, x}^{\frac{3}{2}}\left(\mathbb{R} \times \mathbb{R}^{4}\right)} \leq\left\|e^{i t \Delta} \omega_{n}^{k}\right\|_{W_{1}(\mathbb{R})}^{2}\left\|\nabla e^{i t \Delta} \omega_{n}^{k}\right\|_{V_{0}(\mathbb{R})} .
$$


Then, by the Strichartz estimates and combining the above four inequalities with the fact that $\left\|\omega_{n}^{k}\right\|_{H^{1}\left(\mathbb{R}^{4}\right)} \leq C$, we have

$$
\text { (4.55) } \lesssim\left[\left\|e^{i t \Delta} \omega_{n}^{k}\right\|_{W_{1}(\mathbb{R})}^{2 / 3}+\left\|e^{i t \Delta} \omega_{n}^{k}\right\|_{W_{1}(\mathbb{R})}^{2}\right]
$$

Hence, by the property (4.4) of $\omega_{n}^{k}$, we obtain that (4.55) tends to 0 , as $n, k \rightarrow \infty$.

From the same method of (4.55) and the fact

$$
\sup _{n \geq N_{k}}\left\|\sum_{j=1}^{k} \nabla u_{(n)}^{j}\right\|_{V_{0}(\mathbb{R})} \lesssim B
$$

in (4.26) from Lemma 4.5, we can deal with (4.56).

To deal with (4.57), we consider the terms having the form of

$$
\left\|\left.\left.\left.\left|\sum_{j=1}^{k} u_{(n)}^{j}\right|^{4 / 3} \cdot|| \nabla\right|^{s} e^{i t \Delta} \omega_{n}^{k}\left|\left\|_{L_{t, x}^{\frac{3}{2}}\left(\mathbb{R} \times \mathbb{R}^{4}\right)}+\right\|\right| \sum_{j=1}^{k} u_{(n)}^{j}\right|^{2} \cdot|| \nabla\right|^{s} e^{i t \Delta} \omega_{n}^{k} \mid\right\|_{L_{t, x}^{\frac{3}{2}}\left(\mathbb{R} \times \mathbb{R}^{4}\right)},
$$

where $s \in\{0,1\}$.

By the Hölder inequality and (4.26) in Lemma 4.5) we have

$$
\begin{aligned}
& \left\|\left.\left|\sum_{j=1}^{k} u_{(n)}^{j}\right|^{4 / 3} \cdot|| \nabla\right|^{s} e^{i t \Delta} \omega_{n}^{k} \mid\right\|_{L_{t, x}^{\frac{3}{2}}\left(\mathbb{R} \times \mathbb{R}^{4}\right)} \\
\lesssim & \left\|\sum_{j=1}^{k} u_{(n)}^{j}\right\|_{W_{2}(\mathbb{R})}^{1 / 3} \cdot\left\|\sum_{j=1}^{k} u_{(n)}^{j}|\nabla|^{s} e^{i t \Delta} \omega_{n}^{k}\right\|_{L_{t, x}^{12 / 7}\left(\mathbb{R} \times \mathbb{R}^{4}\right)} \\
\lesssim & \left\|\sum_{j=1}^{k} u_{(n)}^{j}|\nabla|^{s} e^{i t \Delta} \omega_{n}^{k}\right\|_{L_{t, x}^{12 / 7}\left(\mathbb{R} \times \mathbb{R}^{4}\right)^{\prime}}
\end{aligned}
$$

and

$$
\begin{aligned}
& \left\|\left|\sum_{j=1}^{k} u_{(n)}^{j}\right|^{2} \cdot|| \nabla||^{s} e^{i t \Delta} \omega_{n}^{k} \mid\right\|_{L_{t, x}^{\frac{3}{2}}\left(\mathbb{R} \times \mathbb{R}^{4}\right)} \\
\lesssim & \left\|\sum_{j=1}^{k} u_{(n)}^{j}\right\|_{W_{1}(\mathbb{R})} \cdot\left\|\sum_{j=1}^{k} u_{(n)}^{j}|\nabla|^{s} e^{i t \Delta} \omega_{n}^{k}\right\|_{L_{t, x}^{2}\left(\mathbb{R} \times \mathbb{R}^{4}\right)} \\
\lesssim & \left\|\sum_{j=1}^{k} u_{(n)}^{j}|\nabla|^{s} e^{i t \Delta} \omega_{n}^{k}\right\|_{L_{t, x}^{2}\left(\mathbb{R} \times \mathbb{R}^{4}\right)} \cdot
\end{aligned}
$$

Hence, to prove (4.51), we just need show

$$
\lim _{k \rightarrow \infty} \lim _{n \rightarrow \infty}\left\|\sum_{j=1}^{k} u_{(n)}^{j}|\nabla|^{s} e^{i t \Delta} \omega_{n}^{k}\right\|_{L_{t, x}^{p}\left(\mathbb{R} \times \mathbb{R}^{4}\right)}=0
$$

where $p \in\{12 / 7,2\}$ and $s \in\{0,1\}$.

By Lemma 4.4 for any $\varepsilon>0$, there exists $J(\varepsilon)>0$ such that

$$
\sum_{J(\varepsilon) \leq j \leq k}\left\|u_{(n)}^{j}\right\|_{S T(\mathbb{R})}^{2} \leq \sum_{j \geq J(\varepsilon)}\left\|\langle\nabla\rangle U_{\infty}^{j}\right\|_{S(\mathbb{R})}^{2}<\frac{1}{4} \varepsilon^{2} .
$$


Using the same method of proving (4.26), by the almost decoupling condition (4.6), we have can find $N_{k, \varepsilon}>0$ such that: if $n>N_{k, \varepsilon}$, then

$$
\left\|\sum_{J(\varepsilon) \leq j \leq k} u_{(n)}^{j}\right\|_{S T(\mathbb{R})} \leq\left[\sum_{J(\varepsilon) \leq j \leq k}\left\|u_{(n)}^{j}\right\|_{S T(\mathbb{R})}^{2}\right]^{1 / 2}+\frac{1}{2} \varepsilon<\varepsilon,
$$

where in the last step, we used the boundedness of $\left\|\varphi^{j}\right\|_{H^{1}}$.

Then, for $p \in\{12 / 7,2\}$ and $s \in\{0,1\}$, by the Hölder inequality and the Strichartz estimates, we have

$\left\|\sum_{J(\varepsilon) \leq j \leq k} u_{(n)}^{j}|\nabla|^{s} e^{i t \Delta} \omega_{n}^{k}\right\|_{L_{t, x}^{p}\left(\mathbb{R} \times \mathbb{R}^{4}\right)} \leq\left\|\sum_{J(\varepsilon) \leq j \leq k} u_{(n)}^{j}\right\|_{S T(\mathbb{R})}\left\||\nabla|^{s} e^{i t \Delta} \omega_{n}^{k}\right\|_{V_{0}(\mathbb{R})} \lesssim \varepsilon$.

On the other hand, for $p \in\{12 / 7,2\}$ and $s \in\{0,1\}$, by (4.27) in Lemma 4.5. we have

$$
\lim _{k \rightarrow \infty} \lim _{n \rightarrow \infty}\left\|\sum_{1 \leq j \leq J(\varepsilon)} u_{(n)}^{j}|\nabla|^{s} e^{i t \Delta} \omega_{n}^{k}\right\|_{L_{t, x}^{p}\left(\mathbb{R} \times \mathbb{R}^{4}\right)}=0,
$$

which together with (4.69) implies (4.66). This finishes the proof of (4.51).

Proof of (4.52). Recall that $u_{(n)}^{j}$ satisfy that (4.17) and (4.18), then we have

$$
\begin{aligned}
\text { (4.52) } \lesssim & \sum_{s=0,1}\left\||\nabla|^{s}\left[\sum_{j=1}^{k} f\left(u_{(n)}^{j}\right)-f\left(\sum_{j=1}^{k} u_{(n)}^{j}\right)\right]\right\|_{L_{t, x}^{3 / 2}\left(\mathbb{R} \times \mathbb{R}^{4}\right)} \\
& +\sum_{s=0,1}\left\||\nabla|^{s} \sum_{\substack{1 \leq j \leq k \\
h_{n}^{j} \rightarrow 0}}\left[\left(\sigma_{\infty}^{j}\right)^{-1} f_{1}\left(\sigma_{\infty}^{j} u_{(n)}^{j}\right)-f_{1}\left(u_{(n)}^{j}\right)\right]\right\|_{L_{t, x}^{3 / 2}\left(\mathbb{R} \times \mathbb{R}^{4}\right)} \\
& +\sum_{s=0,1}\left\||\nabla|^{s} \sum_{\substack{1 \leq j \leq k \\
h_{n}^{j} \rightarrow 0}} f_{2}\left(u_{(n)}^{j}\right)\right\|_{L_{t, x}^{3 / 2}\left(\mathbb{R} \times \mathbb{R}^{4}\right)}
\end{aligned}
$$

For (4.71), using a elementary inequality, (4.71) can be estimated by

$$
\sum_{s=0,1} \sum_{\substack{1 \leq j, j^{\prime} \leq k \\ j \neq j^{\prime}}}\left\|\left|u_{(n)}^{j}\right|^{2}|\nabla|^{s} u_{(n)}^{j^{\prime}}\right\|_{L_{t, x}^{3 / 2}\left(\mathbb{R} \times \mathbb{R}^{4}\right)}
$$

plus

$$
\sum_{s=0,1} \sum_{\substack{\leq j, j^{\prime} \leq k \\ j \neq j^{\prime}}}\left\|\left|u_{(n)}^{j}\right|^{4 / 3}|\nabla|^{s} u_{(n)}^{j^{\prime}}\right\|_{L_{t, x}^{3 / 2}\left(\mathbb{R} \times \mathbb{R}^{4}\right)} .
$$

For (4.74), we use density. By

$$
\left\|\sigma_{n}^{j} U_{\infty}^{j}-\sigma_{\infty}^{j} U_{\infty}^{j}\right\|_{W_{1}(\mathbb{R})} \lesssim\left\|\frac{\min \left(1-h_{n}^{j}, h_{n}^{j}\right)}{h_{n}^{j}+|\nabla|}\langle\nabla\rangle U_{\infty}^{j}\right\|_{S(\mathbb{R})} \rightarrow 0
$$

and

$$
\left\|\nabla\left[\sigma_{n}^{j} U_{\infty}^{j}-\sigma_{\infty}^{j} U_{\infty}^{j}\right]\right\|_{V_{0}(\mathbb{R})} \lesssim\left\|\frac{\min \left(1-h_{n}^{j}, h_{n}^{j}\right)}{h_{n}^{j}+|\nabla|}\langle\nabla\rangle U_{\infty}^{j}\right\|_{S(\mathbb{R})} \rightarrow 0
$$


we have for any $\varepsilon>0$, there exists $U^{j}, V^{j} \in C_{c}^{\infty}\left(\mathbb{R} \times \mathbb{R}^{4}\right)$ such that for sufficiently large $n$,

$$
\left\|\left[T_{n}^{j} \sigma_{n}^{j} U_{\infty}^{j}-T_{n}^{j} U^{j}\right]\left(\frac{t-t_{n}^{j}}{\left(h_{n}^{j}\right)^{2}}\right)\right\|_{W_{1}(\mathbb{R})}+\left\|\nabla\left[T_{n}^{j} \sigma_{n}^{j} U_{\infty}^{j}-T_{n}^{j} V^{j}\right]\left(\frac{t-t_{n}^{j}}{\left(h_{n}^{j}\right)^{2}}\right)\right\|_{V_{0}(\mathbb{R})}<\varepsilon,
$$

by density. Hence

$$
\text { (4.74) } \lesssim \varepsilon+\sum_{s=0,1} \sum_{\substack{1 \leq j, j^{\prime} \leq k \\ j \neq j^{\prime}}}\left\|\left|T_{n}^{j} U^{j}\right|^{2}\left(\frac{t-t_{n}^{j}}{\left(h_{n}^{j}\right)^{2}}\right) \cdot|\nabla|^{s} T_{n}^{j} V^{j^{\prime}}\left(\frac{t-t_{n}^{j^{\prime}}}{\left(h_{n}^{j^{\prime}}\right)^{2}}\right)\right\|_{L_{t, x}^{3 / 2}\left(\mathbb{R} \times \mathbb{R}^{4}\right)} .
$$

If $\frac{h_{n}^{j}}{h_{n}^{j^{\prime}}} \rightarrow \infty$, we have

$$
\begin{aligned}
& \left\|\left|T_{n}^{j} U^{j}\right|^{2}\left(\frac{t-t_{n}^{j}}{\left(h_{n}^{j}\right)^{2}}\right) \cdot|\nabla| T_{n}^{j^{\prime}} V^{j^{\prime}}\left(\frac{t-t_{n}^{j^{\prime}}}{\left(h_{n}^{j^{\prime}}\right)^{2}}\right)\right\|_{L_{t, x}^{3 / 2}\left(\mathbb{R} \times \mathbb{R}^{4}\right)} \\
\lesssim & \left(h_{n}^{j^{\prime}}\right)^{2}\left(h_{n}^{j}\right)^{-2}\left\|\left|U^{j}\right|^{2}\left(\frac{t\left(h_{n}^{j^{\prime}}\right)^{2}+t_{n}^{j^{\prime}}-t_{n}^{j}}{\left(h_{n}^{j}\right)^{2}}, \frac{x h_{n}^{j^{\prime}}+x_{n}^{j^{\prime}}-x_{n}^{j}}{h_{n}^{j}}\right) \cdot\left[|\nabla| V^{j^{\prime}}\right](t, x)\right\|_{L_{t, x}^{3 / 2}\left(\mathbb{R} \times \mathbb{R}^{4}\right)} \\
\lesssim & \left(h_{n}^{j^{\prime}}\right)^{2}\left(h_{n}^{j}\right)^{-2}\left\|U^{j}\right\|_{L_{t, x}^{\infty}\left(\mathbb{R} \times \mathbb{R}^{4}\right)}\left\||\nabla| V^{j^{\prime}}\right\|_{L_{t, x}^{3 / 2}\left(\mathbb{R} \times \mathbb{R}^{4}\right)}^{2} \rightarrow 0,
\end{aligned}
$$

as $n \rightarrow \infty$.

If $\frac{h_{n}^{j}}{h_{n}^{j}} \rightarrow 0$, we have

$$
\begin{aligned}
&\left\|\left|T_{n}^{j} U^{j}\right|^{2}\left(\frac{t-t_{n}^{j}}{\left(h_{n}^{j}\right)^{2}}\right) \cdot|\nabla| T_{n}^{j^{\prime}} V^{j^{\prime}}\left(\frac{t-t_{n}^{j^{\prime}}}{\left(h_{n}^{j^{\prime}}\right)^{2}}\right)\right\|_{L_{t, x}^{3 / 2}\left(\mathbb{R} \times \mathbb{R}^{4}\right)} \\
& \lesssim\left(h_{n}^{j}\right)^{2}\left(h_{n}^{j^{\prime}}\right)^{-2}\left\|\left|U^{j}\right|^{2}(t, x) \cdot\left[|\nabla| V^{j^{\prime}}\right]\left(\frac{t\left(h_{n}^{j}\right)^{2}+t_{n}^{j}-t_{n}^{j^{\prime}}}{\left(h_{n}^{j^{\prime}}\right)^{2}}, \frac{x h_{n}^{j}+x_{n}^{j}-x_{n}^{j^{\prime}}}{h_{n}^{j^{\prime}}}\right)\right\|_{L_{t, x}^{3 / 2}\left(\mathbb{R} \times \mathbb{R}^{4}\right)} \\
& \lesssim\left(h_{n}^{j}\right)^{2}\left(h_{n}^{j^{\prime}}\right)^{-2} \rightarrow 0
\end{aligned}
$$

as $n \rightarrow \infty$.

If there exists $C_{j}$ such that $\frac{h_{n}^{j}}{h_{n}^{j^{\prime}}}+\frac{h_{n}^{j^{\prime}}}{h_{n}^{j}}<C_{j}$ for any $n$, then by the almost decoupling condition (4.6), we have

$$
\left|\frac{t_{n}^{j^{\prime}}-t_{n}^{j}}{\left(h_{n}^{j}\right)^{2}}\right|+\left|\frac{x_{n}^{j^{\prime}}-x_{n}^{j}}{h_{n}^{j}}\right| \rightarrow \infty, \text { as } n \rightarrow \infty,
$$

where by symmetry we assume that $j<j^{\prime}$.

Then for sufficiently large $n$, we have

$$
\begin{aligned}
& \left\|\left|T_{n}^{j} U^{j}\right|^{2}\left(\frac{t-t_{n}^{j}}{\left(h_{n}^{j}\right)^{2}}\right) \cdot|\nabla| T_{n}^{j^{\prime}} V^{j^{\prime}}\left(\frac{t-t_{n}^{j^{\prime}}}{\left(h_{n}^{j^{\prime}}\right)^{2}}\right)\right\|_{L_{t, x}^{3 / 2}\left(\mathbb{R} \times \mathbb{R}^{4}\right)} \\
\lesssim & \left(h_{n}^{j^{\prime}}\right)^{2}\left(h_{n}^{j}\right)^{-2}\left\|\left|U^{j}\right|^{2}\left(\frac{t\left(h_{n}^{j^{\prime}}\right)^{2}+t_{n}^{j^{\prime}}-t_{n}^{j}}{\left(h_{n}^{j}\right)^{2}}, \frac{x h_{n}^{j^{\prime}}+x_{n}^{j^{\prime}}-x_{n}^{j}}{h_{n}^{j}}\right) \cdot\left[|\nabla| V^{j^{\prime}}\right](t, x)\right\|_{L_{t, x}^{3 / 2}\left(\mathbb{R} \times \mathbb{R}^{4}\right)} \\
= & 0,
\end{aligned}
$$


where we used the fact that $U^{j}, V^{j} \in C_{c}^{\infty}\left(\mathbb{R} \times \mathbb{R}^{4}\right)$.

On the other hand, we need to consider

$$
\sum_{j, j^{\prime}}\left\|\left|u_{(n)}^{j}\right|^{2} u_{(n)}^{j^{\prime}}\right\|_{L_{t, x}^{3 / 2}\left(\mathbb{R} \times \mathbb{R}^{4}\right)} .
$$

If $h_{n}^{j} \equiv 1$, then we have $\left\|\sigma_{n}^{j} U_{\infty}^{j}\right\|_{S(\mathbb{R})}=\left\|U_{\infty}^{j}\right\|_{S(\mathbb{R})} \leq C$. If $h_{n}^{j} \rightarrow 0$, then we have that $\left\|u_{(n)}^{j}\right\|_{V_{0}(\mathbb{R})}=\left(h_{n}^{j}\right)^{2}\left\|\sigma_{n}^{j} U_{\infty}^{j}\right\|_{V_{0}} \lesssim h_{n}^{j}\left\|\langle\nabla\rangle U_{\infty}^{j}\right\|_{S(\mathbb{R})} \rightarrow 0$. Hence by the similar argument as before, we have for any $\varepsilon>0$, there exists $U^{j}, V^{j} \in C_{c}(\mathbb{R} \times \mathbb{R})$ such that

for sufficiently large $n$.

$$
\left\|u_{(n)}^{j}-T_{n}^{j} U^{j}\right\|_{W_{1}(\mathbb{R})}+\left\|u_{(n)}^{j}-T_{n}^{j} V^{j}\right\|_{V_{0}(\mathbb{R})}<\varepsilon
$$

Hence for large $n$, we have

$$
\begin{aligned}
& \sum_{j, j^{\prime}}\left\|\left|u_{(n)}^{j}\right|^{2} u_{(n)}^{j^{\prime}}\right\|_{L^{3 / 2}\left(\mathbb{R} \times \mathbb{R}^{4}\right)} \\
& =\sum_{j, j^{\prime}}\left\|\left|u_{(n)}^{j}\right|^{2}\left[u_{(n)}^{j^{\prime}}-T_{n}^{j^{\prime}} V^{j^{\prime}}\right]\right\|_{L^{3 / 2}\left(\mathbb{R} \times \mathbb{R}^{4}\right)}+\left\|\left|u_{(n)}^{j}\right|^{2} T_{n}^{j^{\prime}} V^{j^{\prime}}\right\|_{L^{3 / 2}\left(\mathbb{R} \times \mathbb{R}^{4}\right)} \\
& \lesssim \sum_{j, j^{\prime}}\left\|u_{(n)}^{j}\right\|_{W_{1}(\mathbb{R})}^{2}\left\|u_{(n)}^{j^{\prime}}-T_{n}^{j^{\prime}} V^{j^{\prime}}\right\|_{V_{0}(\mathbb{R})}+\left\|\left|u_{(n)}^{j}\right|^{2} T_{n}^{j^{\prime}} V^{j^{\prime}}\right\|_{L^{3 / 2}\left(\mathbb{R} \times \mathbb{R}^{4}\right)} \\
& \lesssim \varepsilon+\sum_{j, j^{\prime}}\left(h_{n}^{j^{\prime}}\right)^{3}\left(h_{n}^{j}\right)^{-2}\left\|\left|\sigma_{n}^{j} U_{\infty}^{j}\right|^{2}\left(\frac{t\left(h_{n}^{j^{\prime}}\right)^{2}+t_{n}^{j^{\prime}}-t_{n}^{j}}{\left(h_{n}^{j}\right)^{2}}, \frac{x h_{n}^{j^{\prime}}+x_{n}^{j^{\prime}}-x_{n}^{j}}{h_{n}^{j}}\right) V^{j^{\prime}}(t, x)\right\|_{L^{3 / 2}\left(\mathbb{R} \times \mathbb{R}^{4}\right)} \\
& \lesssim\left(1+h_{n}^{j^{\prime}}\right) \varepsilon+\left(h_{n}^{j^{\prime}}\right)^{3}\left(h_{n}^{j}\right)^{-2}\left\|\left|U^{j}\right|^{2}\left(\frac{t\left(h_{n}^{j^{\prime}}\right)^{2}+t_{n}^{j^{\prime}}-t_{n}^{j}}{\left(h_{n}^{j}\right)^{2}}, \frac{x h_{n}^{j^{\prime}}+x_{n}^{j^{\prime}}-x_{n}^{j}}{h_{n}^{j}}\right) V^{j^{\prime}}(t, x)\right\|_{L^{3 / 2}\left(\mathbb{R} \times \mathbb{R}^{4}\right)} \\
& \lesssim \varepsilon,
\end{aligned}
$$

where in the last inequality we used the almost decoupling condition (4.6) and the fact that $U^{j} \in C_{c}^{\infty}\left(\mathbb{R} \times \mathbb{R}^{4}\right)$.

Note that

$$
\left\|u_{(n)}^{j}\right\|_{W_{2}(\mathbb{R})}+\left\|\langle\nabla\rangle u_{(n)}^{j}\right\|_{V_{0}(\mathbb{R})} \lesssim\left\|\langle\nabla\rangle U_{\infty}^{j}\right\|_{S(\mathbb{R})} .
$$

Hence, we can use the similar argument to prove that (4.75) tends to 0 as $n \rightarrow \infty$.

We consider the contribution of (4.72), by the analogue estimates in Lemma 8.9 of [4]. Indeed, if $j$ such that $h_{n}^{j} \rightarrow 0$, then we have

$$
\begin{aligned}
& \left\|\langle\nabla\rangle\left[\left(\sigma_{\infty}^{j}\right)^{-1} f_{1}\left(\sigma_{\infty}^{j} u_{(n)}^{j}\right)-f_{1}\left(u_{(n)}^{j}\right)\right]\right\|_{L_{t, x}^{3 / 2}\left(\mathbb{R} \times \mathbb{R}^{4}\right)} \\
= & \left\||\nabla| f_{1}\left(\sigma_{\infty}^{j} U_{\infty}^{j}\right)-\left(h_{n}^{j}+|\nabla|\right) f_{1}\left(\sigma_{n}^{j} U_{\infty}^{j}\right)\right\|_{L_{t, x}^{3 / 2}\left(\mathbb{R} \times \mathbb{R}^{4}\right)} \\
\lesssim & \left\|\left|\sigma_{\infty}^{j} U_{\infty}^{j}\right|^{2}\langle\nabla\rangle U_{\infty}^{j}-\left|\sigma_{n}^{j} U_{\infty}^{j}\right|^{2}\langle\nabla\rangle U_{\infty}^{j}\right\|_{L_{t, x}^{3 / 2}\left(\mathbb{R} \times \mathbb{R}^{4}\right)} \\
\lesssim & \left\|\langle\nabla\rangle U_{\infty}^{j}\right\|_{S(\mathbb{R})}\left\|\sigma_{\infty}^{j} U_{\infty}^{j}\right\|_{W_{1}(\mathbb{R})}\left\|\sigma_{\infty}^{j} U_{\infty}^{j}-\sigma_{n}^{j} U_{\infty}^{j}\right\|_{W_{1}(\mathbb{R})} \\
\lesssim & \left\|\frac{h_{n}^{j}}{h_{n}^{j}+|\nabla|}\langle\nabla\rangle U_{\infty}^{j}\right\|_{W_{1}(\mathbb{R})} \rightarrow 0,
\end{aligned}
$$

as $n \rightarrow \infty$, which implies 4.72) $\rightarrow 0$. 
For (4.73), we have

$$
\begin{aligned}
\text { (4.73) } & \lesssim \sum_{\substack{1 \leq j \leq k \\
h_{n}^{j} \rightarrow 0}}\left(h_{n}^{j}\right)^{2 / 3}\left\|\langle\nabla\rangle f_{2}\left(\sigma_{n}^{j} U_{\infty}^{j}\right)\right\|_{L_{t, x}^{3 / 2}\left(\mathbb{R} \times \mathbb{R}^{4}\right)} \\
& \lesssim \sum_{\substack{1 \leq j \leq k \\
h_{n}^{j} \rightarrow 0}}\left(h_{n}^{j}\right)^{2 / 3}\left\|\sigma_{n}^{j} U_{\infty}^{j}\right\|_{W_{2}(\mathbb{R})}^{4 / 3}\left[\left\||\nabla| \sigma_{n}^{j} U_{\infty}^{j}\right\|_{V_{0}(\mathbb{R})}+h_{n}^{j}\left\|\sigma_{n}^{j} U_{\infty}^{j}\right\|_{V_{0}(\mathbb{R})}\right] \\
& \lesssim \sum_{\substack{1 \leq j \leq k \\
h_{n}^{j} \rightarrow 0}}\left\|\frac{\left(h_{n}^{j}\right)^{1 / 2}|\nabla|^{1 / 2}}{h_{n}^{j}+|\nabla|}\langle\nabla\rangle U_{\infty}^{j}\right\|_{V_{2}(\mathbb{R})}^{4 / 3}\left\|\langle\nabla\rangle U_{\infty}^{j}\right\|_{V_{0}(\mathbb{R})} \rightarrow 0
\end{aligned}
$$

as $n \rightarrow \infty$, which ends the proof of (4.52).

\section{GWP AND SCATTERING OF $\mathcal{K}^{+}$}

In this part, we will prove Theorem 1.1 by the argument of contradiction.

Firstly, we define the the minimal blowup energy of (CNLS). For fixed $C \in$ $(0, \infty)$, and any $E \in(0, \infty)$, let

$$
A_{C}(E)=\sup \left\{\|u\|_{S T(I)}: u_{0} \in \mathcal{K}^{+} \text {and } E\left(u_{0}\right) \leq E, M\left(u_{0}\right) \leq C\right\},
$$

where $u$ is the solution to (CNLS) on the maximal lifespan time interval $I$. Suppose $E_{C}^{*}$ such that $A_{C}\left(E_{C}^{*}\right)=\infty$ is the minimal blowup energy.

Next, we will prove that: If $E_{C}^{*}<m$, then there exists a critical element with energy equal to $E_{C}^{*}$ and has some special properties.

Finally, by making use of the interaction Morawetz estimates similar in [13, we will prove that the critical element equal to zero, which contradicts with the small data theory.

\subsection{Existence of the critical element.}

Lemma 5.1. Let $\left\{u_{n}\right\}$ be a sequence of solutions of (CNLS) in $\mathcal{K}^{+}$on $I_{n} \subset \mathbb{R}$ satisfying $M\left(u_{n}\right) \leq C$ and

$$
E\left(u_{n}\right) \rightarrow E_{C}^{*}<m,\left\|u_{n}\right\|_{S T\left(I_{n}\right)} \rightarrow \infty \text { as } n \rightarrow \infty .
$$

Then there exists a global solution $u_{c}$ of $(C N L S)$ in $\mathcal{K}^{+}$satisfying

$$
E\left(u_{c}\right)=E_{C}^{*}<m,\left\|u_{c}\right\|_{S T(\mathbb{R})}=\infty .
$$

In addition, there exist a sequence $\left(t_{n}, x_{n}\right) \in\left(\mathbb{R} \times \mathbb{R}^{4}\right)$ and $\varphi \in H^{1}\left(\mathbb{R}^{4}\right)$ such that, up to a subsequence, we have as $n \rightarrow \infty$,

$$
\left\|u_{n}(0, x)-e^{-i t_{n} \Delta} \varphi\left(x-x_{n}\right)\right\|_{\dot{H}^{1}\left(\mathbb{R}^{4}\right)} \rightarrow 0 .
$$

Proof. For the sequence $\left\{u_{n}\right\}$ satisfying (5.2), by the time translation symmetry of (CNLS) we can assume that for each $n, 0 \in I_{n}$. As the discussion in Section 4, we have the linear and nonlinear profile decomposition

$$
e^{i t \Delta} u_{n}(0, x)=\sum_{j=1}^{k} v_{n}^{j}(t, x)+e^{i t \Delta} \omega_{n}^{k}, v_{n}^{j}(t, x)=e^{i\left(t-t_{n}^{j}\right) \Delta} T_{n}^{j} \sigma_{n}^{j} \varphi^{j} ;
$$




$$
\begin{array}{r}
\tilde{u}_{n}^{k} \triangleq \sum_{j=1}^{k} u_{(n)}^{j}+e^{i t \Delta} \omega_{n}^{k}, u_{(n)}^{j}(t, x)=T_{n}^{j} \sigma_{n}^{j} U_{\infty}^{j}\left(\frac{t-t_{n}^{j}}{\left(h_{n}^{j}\right)^{2}}\right) ; \\
\left\|u_{(n)}^{j}(0)-v_{n}^{j}(0)\right\|_{H^{1}\left(\mathbb{R}^{4}\right)} \rightarrow 0, \text { as } n \rightarrow \infty .
\end{array}
$$

By Proposition 4.6, we have that there exists at least one profile $j_{0}$ such that $\left\|\sigma_{\infty}^{j_{0}} U_{\infty}^{j_{0}}\right\|_{S T^{j_{0}\left(I^{j_{0}}\right)}}=\infty$.

Moreover, we claim that there is only one profile in the profile decomposition. In fact, this follows from the definition of $E_{C}^{*}$ and the fact that every solution of (CNLS) in $\mathcal{K}^{+}$with energy less than $E_{C}^{*}$ has global finite Strichartz norm.

In summary, we have

$$
E\left(u_{(n)}^{1}(0)\right) \rightarrow E_{C}^{*}, \quad u_{(n)}^{1} \in \mathcal{K}^{+}, \quad\left\|\sigma_{\infty}^{1} U_{\infty}^{1}\right\|_{S T^{1}\left(I_{1}\right)}=\infty \text { and }\left\|\omega_{n}^{1}\right\|_{\dot{H}^{1}\left(\mathbb{R}^{4}\right)} \rightarrow 0 .
$$

If $h_{n}^{1} \rightarrow 0$, then we have $\sigma_{\infty}^{1} U_{\infty}^{1}$ satisfies $i \partial_{t} u+\Delta u+|u|^{2} u=0$ and

$$
E_{c}\left(\sigma_{\infty}^{1} U_{\infty}^{1}\right)=E_{C}^{*}<m, \quad\left\|\sigma_{\infty}^{1} U_{\infty}^{1}\right\|_{\dot{H}^{1}}<\|W\|_{\dot{H}^{1}}, \quad\left\|\sigma_{\infty}^{1} U_{\infty}^{1}\right\|_{W_{1}\left(I_{1}\right)}=\infty,
$$

which contradicts with the results in 13 .

Hence we have $h_{n}^{0} \equiv 1$. Let $u_{c}=U_{\infty}^{1}$, which is a solution of (CNLS) and satisfies $E\left(u_{c}\right)=E_{C}^{*}<m$, which implies $u_{c} \in \mathcal{K}^{+}$. (5.4) follows from the linear profile decomposition and the fact that $\left\|\omega_{n}^{1}\right\|_{\dot{H}^{1}\left(\mathbb{R}^{4}\right)} \rightarrow 0$.

To prove (5.3), we only need to prove $u_{c}$ is a global solution. If not, we can choose a sequence $t_{n}$ tends to the finite boundary of $I_{1}$. Then we have $u_{c}\left(t+t_{n}\right)$ with finite mass and satisfying the condition (5.2). Hence, by the above discussion, we can find a sequence $\left(t_{n}^{\prime}, x_{n}^{\prime}\right)$ and a function $\phi \in H^{1}\left(\mathbb{R}^{4}\right)$, such that

$$
\left\|u_{c}\left(t_{n}\right)-e^{-i t_{n}^{\prime} \Delta} \phi\left(x-x_{n}^{\prime}\right)\right\|_{\dot{H}^{1}\left(\mathbb{R}^{4}\right)} \rightarrow 0, \text { as } n \rightarrow \infty .
$$

For any $\varepsilon>0$, by the Strichartz estimates, we can find $\delta>0$, such that

$$
\left\|e^{i t \Delta} \phi\right\|_{W_{1}\left(\left[t_{n}-t_{n}^{\prime}-\delta, t_{n}-t_{n}^{\prime}+\delta\right]\right)}+\left\|\nabla e^{i t \Delta} \phi\right\|_{L_{t, x}^{3}\left(\left[t_{n}-t_{n}^{\prime}-\delta, t_{n}-t_{n}^{\prime}+\delta\right]\right)}<\varepsilon .
$$

This and (5.6) imply that

$$
\left\|e^{i t \Delta} u_{c}\left(t_{n}\right)\right\|_{W_{1}([-\delta, \delta])}+\left\|\nabla e^{i t \Delta} u_{c}\left(t_{n}\right)\right\|_{L_{t, x}^{3}([-\delta, \delta])}<\varepsilon,
$$

for sufficiently large $n$ (up to a subsequence). Then by the Strichartz estimates and the Hölder inequality, we have

$$
\begin{aligned}
& \left\|u_{c}(t)\right\|_{W_{1}\left(\left[t_{n}-\delta, t_{n}+\delta\right]\right)}+\left\|\nabla u_{c}(t)\right\|_{L_{t, x}^{3}\left(\left[t_{n}-\delta, t_{n}+\delta\right]\right)} \\
\lesssim & +\left\|\nabla\left[f_{1}\left(u_{c}(t)\right)+f_{2}\left(u_{c}(t)\right)\right]\right\|_{L_{t, x}^{3 / 2}\left(\left[t_{n}-\delta, t_{n}+\delta\right]\right)} \\
\lesssim & \varepsilon+\left\|u_{c}(t)\right\|_{W_{1}\left(\left[t_{n}-\delta, t_{n}+\delta\right]\right)}^{2}\left\|\nabla u_{c}(t)\right\|_{V_{0}\left(\left[t_{n}-\delta, t_{n}+\delta\right]\right)} \\
& +\left\|u_{c}(t)\right\|_{W_{1}\left(\left[t_{n}-\delta, t_{n}+\delta\right]\right)}^{\frac{4}{3}}\left\|\nabla u_{c}(t)\right\|_{V_{0}\left(\left[t_{n}-\delta, t_{n}+\delta\right]\right)}^{1 / 3}\left\|\nabla u_{c}(t)\right\|_{L_{t, x}^{2}\left(\left[t_{n}-\delta, t_{n}+\delta\right]\right)}^{2 / 3} .
\end{aligned}
$$

By the standard continuous method and the fact that $u_{c} \in \mathcal{K}^{+}$, we have that $\left\|u_{c}(t)\right\|_{W_{1}\left(\left[t_{n}-\delta, t_{n}+\delta\right]\right)}<+\infty$.

On the other hand, by Sobolev embedding relation $\dot{H}^{1}\left(\mathbb{R}^{4}\right) \hookrightarrow L^{4}\left(\mathbb{R}^{4}\right)$, we have that $\left\|u_{c}(t)\right\|_{W_{2}\left(\left[t_{n}-\delta, t_{n}+\delta\right]\right)}<+\infty$. Hence $\left\|u_{c}\right\|_{S T\left(\left[t_{n}-\delta, t_{n}+\delta\right]\right)}<+\infty$, which contradicts with the fact that $u_{c}$ is not a global solution. 
By the local well-posedness theory, if we take $\varepsilon$ small enough, the solution $u_{c}(t)$ exists on $\left[t_{n}-\delta, t_{n}+\delta\right]$, which contradicts with the choice of $t_{n}$. Hence we have $u_{c}$ is a global solution.

5.2. Compactness of the critical element. In this subsection, for the critical elements as obtained above, we give out their compactness property and an important corollary.

Proposition 5.2. Let $u_{c}$ be a forward critical element, i.e.

$$
\left\|u_{c}\right\|_{S T([0, \infty))}=\infty .
$$

Then there exists $x(t):(0, \infty) \rightarrow \mathbb{R}^{4}$ such that the set

$$
\left\{u_{c}(t, x-x(t)): 0<t<\infty\right\}
$$

is precompact in $\dot{H}^{s}$ for any $s \in(0,1]$.

Proof. By the observation that the mass of $u_{c}$ conserves of time, we just need to prove the precompacness property in the space $\dot{H}^{1}\left(\mathbb{R}^{4}\right)$.

To do this, for any sequence $\left\{t_{n}\right\} \subset[0, \infty)$, we need find a sequence $\left\{x_{n}\right\} \in \mathbb{R}^{4}$ such that the set $\left\{u_{c}\left(t_{n}, x+x_{n}\right)\right\}$ is precompact in $\dot{H}^{1}\left(\mathbb{R}^{4}\right)$. If there exists a subsequence $\left\{t_{n_{k}}\right\}$ of $\left\{t_{n}\right\}$ converges, the claim follows from the time continuousness of $u_{c}$. If $t_{n}$ converges to $\infty$, by profile decomposition and the discussion in Lemma 5.1. there exist a sequence $\left(x_{n}, t_{n}^{\prime}\right) \in \mathbb{R}^{4} \times[0, \infty)$ and a function $\varphi \in H^{1}$ such that

$$
\left\|u_{c}\left(t_{n}\right)-e^{-i t_{n}^{\prime} \Delta} \varphi\left(x-x_{n}\right)\right\|_{\dot{H}^{1}\left(\mathbb{R}^{4}\right)} \rightarrow 0, \text { as } n \rightarrow \infty .
$$

Hence we will be done if $t_{n}^{\prime}$ converges to a constant. Thus we just need to preclude the two cases below:

(1) If $t_{n}^{\prime} \rightarrow-\infty$, by the Strichartz estimates, we have

$$
\begin{aligned}
& \left\|e^{i t \Delta} u_{c}\left(t_{n}\right)\right\|_{S T([0, \infty))}+\left\|\nabla e^{i t \Delta} u_{c}\left(t_{n}\right)\right\|_{L_{t, x}^{3}([0, \infty))}+\left\||\nabla|^{1 / 2} e^{i t \Delta} u_{c}\left(t_{n}\right)\right\|_{L_{t, x}^{3}([0, \infty))} \\
\lesssim & \left\|\langle\nabla\rangle e^{i t \Delta} \varphi\right\|_{S\left(\left[-t_{n}^{\prime}, \infty\right)\right)}+\left\|u_{c}\left(t_{n}\right)-e^{-i t_{n}^{\prime} \Delta} \varphi\left(x-x_{n}\right)\right\|_{\dot{H}^{1} \cap \dot{H}^{1 / 2}\left(\mathbb{R}^{4}\right)} .
\end{aligned}
$$

Notice that the mass of $u_{c}$ and $e^{-i t_{n}^{\prime} \Delta} \varphi\left(x-x_{n}\right)$ is bounded, which together with interpolation implies $\dot{H}^{1 / 2}\left(\mathbb{R}^{4}\right)$ norm of their difference also tends to 0 . Hence for any $\varepsilon>0$, by Strichartz estimates and the fractional chain rule, we have

$$
\begin{aligned}
& \left\|u_{c}\right\|_{L_{t, x}^{6}\left(\left[t_{n}, \infty\right)\right)}+\left\|u_{c}\right\|_{L_{t, x}^{4}\left(\left[t_{n}, \infty\right)\right)}+\left\|\nabla u_{c}\right\|_{L_{t, x}^{3}\left(\left[t_{n}, \infty\right)\right)}+\left\||\nabla|^{1 / 2} u_{c}\right\|_{L_{t, x}^{3}\left(\left[t_{n}, \infty\right)\right)} \\
& \lesssim \varepsilon+\left\|u_{c}\right\|_{L_{t, x}^{6}\left(\left[t_{n}, \infty\right)\right)}^{2}\left[\left\|\nabla u_{c}\right\|_{L_{t, x}^{3}\left(\left[t_{n}, \infty\right)\right)}+\left\||\nabla|^{1 / 2} u_{c}\right\|_{L_{t, x}^{3}\left(\left[t_{n}, \infty\right)\right)}\right] \\
& +\left\|u_{c}\right\|_{L_{t, x}^{4}\left(\left[t_{n}, \infty\right)\right)}^{4 / 3}\left[\left\|\nabla u_{c}\right\|_{L_{t, x}^{3}\left(\left[t_{n}, \infty\right)\right)}+\left\||\nabla|^{1 / 2} u_{c}\right\|_{L_{t, x}^{3}\left(\left[t_{n}, \infty\right)\right)}\right],
\end{aligned}
$$

by taking $n$ sufficiently large. By the standard continuous method, we can find that $\left\|u_{c}\right\|_{S T\left(\left[t_{n}, \infty\right)\right)}<+\infty$, which contradicts with (5.8).

(2) If $t_{n}^{\prime} \rightarrow+\infty$, then we have

$$
\begin{aligned}
& \left\|e^{i t \Delta} u_{c}\left(t_{n}\right)\right\|_{S T((-\infty, 0))}+\left\|\nabla e^{i t \Delta} u_{c}\left(t_{n}\right)\right\|_{L_{t, x}^{3}([0, \infty))}+\left\||\nabla|^{1 / 2} e^{i t \Delta} u_{c}\left(t_{n}\right)\right\|_{L_{t, x}^{3}([0, \infty))} \\
\lesssim & \left\|\langle\nabla\rangle e^{i t \Delta} \varphi\right\|_{S\left(\left(-\infty,-t_{n}^{\prime}\right)\right)}+\left\|u_{c}\left(t_{n}\right)-e^{-i t_{n}^{\prime} \Delta} \varphi\left(x-x_{n}\right)\right\|_{\dot{H}^{1} \cap \dot{H}^{1 / 2}\left(\mathbb{R}^{4}\right)} \rightarrow 0 .
\end{aligned}
$$


MIAO, ZHAO, AND ZHENG

By a similar way as in (1), we have $\left\|u_{c}\right\|_{S T\left(-\infty, t_{n}\right)} \rightarrow 0$, which implies $u_{c}=0$ and contradicts with (5.8).

By Proposition 5.2 and the Arzela-Ascoli theorem, we have

Corollary 5.3. Let $u_{c}(t, x)$ be a critical element, then there exists $x(t):(0, \infty) \rightarrow$ $\mathbb{R}^{4}$ such that: for any $\eta>0$, there exists $C(\eta)<\infty$ such that

$\int_{|x-x(t)|>C(\eta)}|\nabla u(t, x)|^{2} d x+\int_{|\xi|>C(\eta)}|\xi|^{2}|\hat{u}(t, \xi)|^{2} d \xi+\int_{|\xi|<\frac{1}{C(\eta)}}|\xi|^{2}|\hat{u}(t, \xi)|^{2} d \xi<\eta$

5.3. Extinction of the critical element. In this part, we will finish the proof of the main theorem. We will use the interaction Morawetz estimate to exclude the critical element in Proposition 5.2 .

Theorem 5.4. Suppose $u$ is a critical element in Proposition 5.2 satisfying that

$$
u \in \mathcal{K}^{+}=\left\{u(t) \in H^{1}: E(u)<m, K_{\alpha, \beta}(u) \geq 0,(\alpha, \beta) \in \Omega\right\} .
$$

Then we have $u \equiv 0$.

Proof. Let $\psi \in C_{0}^{\infty}(\mathbb{R})$ be a radial function such that $\psi(x)=1$ when $|x| \leq 1$ and $\psi(x)=0$ when $|x|>2$. Next, let

$$
\phi(x-y)=\int \psi^{2}(x-s) \psi^{2}(y-s) d s .
$$

Hence $\phi(x)$ is supported on $|x| \leq 4$. For $1 \leq R \leq R_{0}$ define the interaction Morawetz potential

$$
M_{R}(t)=\int|u(t, y)|^{2} \phi\left(\frac{|x-y|}{R}\right)(x-y)_{j} \operatorname{Im}\left[\bar{u} \partial_{j} u\right](t, x) d x d y
$$

and let

$$
M(t)=\int_{1 \leq R \leq R_{0}} \frac{1}{R} M_{R}(t) d R .
$$

By the Hölder inequality and the Sobolev embedding theorem, we have

$$
\sup _{t \in \mathbb{R}}\left|M_{R}(t)\right| \lesssim R^{4}
$$

By a direct calculation, we have

$$
\begin{aligned}
& \frac{d}{d t} M_{R}(t) \\
= & 2 \int|u|^{2}(t, y) \phi\left(\frac{|x-y|}{R}\right)\left[|\nabla u|^{2}-|u|^{4}+\frac{4}{5}|u|^{\frac{10}{3}}\right](t, x) d x d y \\
& -2 \int \operatorname{Im}\left[\bar{u} \partial_{j} u\right](t, y) \phi\left(\frac{|x-y|}{R}\right) \operatorname{Im}\left[\bar{u} \partial_{j} u\right](t, x) d x d y \\
& +2 \int|u|^{2}(t, y) \phi^{\prime}\left(\frac{|x-y|}{R}\right) \frac{(x-y)_{j}(x-y)_{k}}{|x-y| R} \operatorname{Re}\left[\left(\partial_{j} \bar{u} \partial_{k} u\right)\right](t, x) d x d y \\
& +\int|u|^{2}(t, y) \phi^{\prime}\left(\frac{|x-y|}{R}\right) \frac{|x-y|}{R}\left[|\nabla u|^{2}-\frac{1}{2}|u|^{4}+\frac{2}{5}|u|^{\frac{10}{3}}\right](t, x) d x d y \\
& -2 \int \operatorname{Im}\left[\bar{u} \partial_{k}\right](t, y) \phi^{\prime}\left(\frac{|x-y|}{R}\right) \frac{(x-y)_{j}(x-y)_{k}}{|x-y| R} \operatorname{Im}\left[\bar{u} \partial_{j} u\right](t, x) d x d y
\end{aligned}
$$




$$
-\frac{1}{2} \int|u|^{2}(t, y) \Delta\left[4 \phi\left(\frac{|x-y|}{R}\right)+\phi^{\prime}\left(\frac{|x-y|}{R}\right) \frac{|x-y|}{R}\right]|u|^{2}(t, x) d x d y .
$$

By Proposition 5.2 and conservation of mass, we have $u(t, x) \in L_{t}^{\infty} H^{1}\left(\mathbb{R}^{4}\right)$. Let $I=(0, K) \subseteq \mathbb{R}$ and $1 \leq R_{0} \leq K^{\frac{1}{5}}$.

First we consider (5.15), (5.16) and (5.17). From the definition of $\phi$, we have

$$
\int_{1 \leq R \leq R_{0}} \frac{1}{R}\left|\phi^{\prime}\left(\frac{|x-y|}{R}\right) \frac{(x-y)_{j}(x-y)_{k}}{|x-y| R}\right| d R \lesssim \int_{1 \leq R \leq R_{0}} \frac{1}{R}\left|\phi^{\prime}\left(\frac{|x-y|}{R}\right)\right| \frac{|x-y|}{R} d R \lesssim 1,
$$

and is supported on $|x-y| \lesssim R_{0}$. Hence we have

$$
\left|\int_{I} \int_{1 \leq R \leq R_{0}} \frac{1}{R}[(\underline{5.15})+(\underline{5.16})+(\underline{5.17})] d R d t\right| \lesssim K .
$$

Next we consider (5.18). Notice that

$$
\begin{aligned}
& \left|\int_{1 \leq R \leq R_{0}} \frac{1}{R} \Delta\left[4 \phi\left(\frac{|x-y|}{R}\right)+\phi^{\prime}\left(\frac{|x-y|}{R}\right) \frac{|x-y|}{R}\right] d R\right| \\
= & \left|\int_{1 \leq R \leq R_{0}} \frac{1}{R}\left[\phi^{\prime \prime \prime}\left(\frac{|x-y|}{R}\right) \frac{|x-y|}{R^{3}}+\phi^{\prime \prime}\left(\frac{|x-y|}{R}\right) \frac{6}{R^{2}}+\phi^{\prime}\left(\frac{|x-y|}{R}\right) \frac{15}{R|x-y|}\right] d R\right| \\
\lesssim & \frac{1}{1+|x-y|^{2}}+\frac{1}{|x-y|+|x-y|^{2}} .
\end{aligned}
$$

This together with the Hardy-Littlewood-Sobolev inequality, the Sobolev theorem and $u \in L_{t}^{\infty} H_{x}^{1}$ implies that

$$
\begin{aligned}
& \int_{I} \int_{1 \leq R \leq R_{0}}(5.18) \frac{1}{R} d R d t \\
& \lesssim \int_{I} \int|u|^{2}(t, x)\left(1+\frac{1}{|x-y|^{2}}\right)|u|^{2}(t, y) d x d y d t \\
& \lesssim K\left(\|u\|_{L_{t}^{\infty} L_{x}^{2}}^{4}+\|u\|_{L_{t}^{\infty} L_{x}^{\frac{8}{3}}}^{4}\right) \\
& \lesssim K \text {. }
\end{aligned}
$$

Now we will estimate (5.13) and (5.14). By abuse of notations, we will write $\psi(x)=\psi(|x|)$, for $x \in \mathbb{R}^{4}$. Notice that for each $s$ and $t$, there exists a $\xi(s, t)$ such that

$$
\begin{aligned}
& \int \psi^{2}\left(\frac{x}{R}-s\right) \operatorname{Im}\left[\overline{e^{i x \cdot \xi(s, t)} u} \nabla\left(e^{i x \cdot \xi(s, t)} u\right)\right](t, x) d x \\
= & \int \psi^{2}\left(\frac{x}{R}-s\right) \operatorname{Im}[\bar{u} \nabla u](t, x) d x+\xi(s, t) \int \psi^{2}\left(\frac{x}{R}-s\right)|u|^{2}(t, x) d x \\
= & 0 .
\end{aligned}
$$

Moreover, for any $s, t$ and $\xi(s, t)$, the quantity

$$
\int \psi^{2}\left(\frac{x}{R}-s\right) \psi^{2}\left(\frac{y}{R}-s\right)\left[|\nabla u(t, x)|^{2}|u(t, y)|^{2}-\operatorname{Im}[\bar{u} \nabla u](t, x) \operatorname{Im}[\bar{u} \nabla u](t, y)\right] d x d y
$$

is invariant under the Galilean transformation $u(t, x) \mapsto e^{i x \cdot \xi(s, t)} u(t, x)$. Therefore, for any given $s, t$ we can choose $\xi(s, t)$ to eliminate the momentum squared terms. 
Then, from integration by parts, we have

$$
\begin{aligned}
& \int \psi^{2}\left(\frac{x}{R}-s\right)\left[\left|\nabla\left(e^{i x \cdot \xi(s, t)} u(t, x)\right)\right|^{2}-|u(t, x)|^{4}\right] d x \\
= & \int\left|\nabla\left(\psi\left(\frac{x}{R}-s\right) e^{i x \cdot \xi(s, t)} u(t, x)\right)\right|^{2} d x-\int \psi^{2}\left(\frac{x}{R}-s\right)|u(t, x)|^{4} d x \\
& +\int|u(t, x)|^{2} \psi\left(\frac{x}{R}-s\right) \Delta_{x} \psi\left(\frac{x}{R}-s\right) d x .
\end{aligned}
$$

Since $u \in \mathcal{K}^{+}$, we have $\|u\|_{L_{t}^{\infty} \dot{H}^{1}} \leq(1-\bar{\delta})\|W\|_{\dot{H}^{1}}$ by Lemma 2.14, Therefore, by the sharp Sobolev inequalities, we have

$$
\|u\|_{L^{4}\left(\mathbb{R}^{4}\right)} \leq \frac{\|\nabla u\|_{L^{2}\left(\mathbb{R}^{4}\right)}}{\|W\|_{L^{4}\left(\mathbb{R}^{4}\right)}} \leq \frac{1}{\|W\|_{L^{4}\left(\mathbb{R}^{4}\right)}}(1-\bar{\delta})\|W\|_{\dot{H}^{1}} \leq(1-\bar{\delta})\|W\|_{L^{4}\left(\mathbb{R}^{4}\right)} .
$$

Let $v=e^{i x \cdot \xi(s, t)} u$, then by Hölder inequalities we have

$$
\begin{aligned}
\text { (5.24) } & \geq\|\nabla(\psi v)\|_{L^{2}}^{2}-(1+\bar{\delta})\left\|\psi^{2}|u|^{4}\right\|_{L^{1}}+\bar{\delta}\left\|\psi^{2}|u|^{4}\right\|_{L^{1}} \\
& \geq\|\nabla(\psi v)\|_{L^{2}}^{2}-(1+\bar{\delta})(1-\bar{\delta})^{2}\|\nabla(\psi v)\|_{L^{2}}^{2}+\bar{\delta}\left\|\psi^{2}|u|^{4}\right\|_{L^{1}} \\
& \geq \bar{\delta}\left\|\psi^{2}|u|^{4}\right\|_{L^{1}} .
\end{aligned}
$$

For (5.25), noting that

$$
\int \psi\left(\frac{x}{R}-s\right)\left|\Delta_{x} \psi\left(\frac{x}{R}-s\right)\right| \cdot \psi^{2}\left(\frac{y}{R}-s\right) d s \lesssim \psi\left(\frac{x-y}{4 R}\right)\left(\frac{1}{R^{2}}+\frac{1}{R|x-y|}\right),
$$

we have

$$
\begin{aligned}
& \left.\left|\int_{I} \int_{1 \leq R \leq R_{0}} \frac{1}{R} \int(5.25)\right| u\right|^{2}(t, y) d y d s d R d t \mid \\
\lesssim & \int_{I} \iint_{1 \leq R \leq R_{0}} \frac{1}{R}|u|^{2}(t, x)\left[\psi\left(\frac{|x-y|}{4 R}\right)\left(\frac{1}{R^{2}}+\frac{1}{R|x-y|}\right)\right]|u|^{2}(t, y) d R d x d y d t \\
\lesssim & \int_{I} \int|u|^{2}(t, x)\left(1+\frac{1}{|x-y|^{2}}\right)|u|^{2}(t, y) d x d y d t \\
\lesssim K &
\end{aligned}
$$

For $|x-y| \leq R_{0}^{\frac{1}{2}}$ and $R \geq R_{0}^{\frac{1}{2}}$, we have

$$
\int \psi^{2}\left(\frac{x}{R}-s\right) \psi^{2}\left(\frac{y}{R}-s\right) d s \gtrsim 1
$$

Thus, we have

$$
\begin{aligned}
& \int_{I} \int_{R_{0}^{\frac{1}{2}} \leq R \leq R_{0}} \frac{1}{R} \int|u|^{2}(t, y) \psi^{2}\left(\frac{x}{R}-s\right) \psi^{2}\left(\frac{y}{R}-s\right)|u|^{4}(t, x) d s d x d y d R d t \\
\gtrsim & \int_{I} \int_{R_{0}^{\frac{1}{2}} \leq R \leq R_{0}} \frac{1}{R} \int_{|x-y| \leq R_{0}^{\frac{1}{2}}} \int|u|^{2}(t, y)|u|^{4}(t, x) d x d y d R d t \\
\gtrsim & \ln R_{0} \int_{I} \int_{|x-y| \leq R_{0}^{\frac{1}{2}}} \int|u|^{2}(t, y)|u|^{4}(t, x) d x d y d t \\
\gtrsim & \ln R_{0} \int_{I}\left[\int_{|x-x(t)| \leq R_{0}^{\frac{1}{4}}}|u|^{4}(t, x) d x \cdot \int_{|y-x(t)| \leq R_{0}^{\frac{1}{4}}}|u|^{2}(t, y) d y\right] d t .
\end{aligned}
$$


This together with (5.12), (5.19) and (5.21) implies

$$
\begin{aligned}
R_{0}^{4} & \gtrsim \int_{1 \leq R \leq R_{0}} \frac{1}{R} \sup _{t}\left|M_{R}((t))\right| d R \\
& \gtrsim\left|\int_{I} \int_{1 \leq R \leq R_{0}} \frac{1}{R} \frac{d}{d t} M_{R}(t) d t d R\right| \\
& \gtrsim \ln R_{0} \int_{I}\left[\int_{|x-x(t)| \leq R_{0}^{\frac{1}{4}}}|u|^{4}(t, x) d x \cdot \int_{|y-x(t)| \leq R_{0}^{\frac{1}{4}}}|u|^{2}(t, y) d y\right] d t-K .
\end{aligned}
$$

Since $1 \leq R_{0} \leq K^{\frac{1}{5}}$, we have

$$
\ln R_{0} \int_{I}\left[\int_{|x-x(t)| \leq R_{0}^{\frac{1}{4}}}|u|^{4}(t, x) d x \cdot \int_{|y-x(t)| \leq R_{0}^{\frac{1}{4}}}|u|^{2}(t, y) d y\right] d t \lesssim K .
$$

Furthermore, (5.33) implies that there exists a sequence $t_{n} \in R$ such that either

or

$$
\int_{\left|x-x\left(t_{n}\right)\right| \leq R_{0, n}^{\frac{1}{4}}}|u|^{4}\left(t_{n}, x\right) d x \rightarrow 0, R_{0, n} \rightarrow \infty
$$

$$
\int_{\left|y-x\left(t_{n}\right)\right| \leq R_{0, n}^{\frac{1}{4}}}|u|^{2}\left(t_{n}, y\right) d y \rightarrow 0, R_{0, n} \rightarrow \infty .
$$

By Corollary 5.3 and the Sobolev inequalities, (5.34) and (5.35) implies $u \equiv 0$.

Acknowledgments:The authors thank the referee and the associated editor for their invaluable comments and suggestions which helped improve the paper greatly. This work was supported in part by the National Natural Science Foundation of China under grants 11671047.

\section{REFERENCES}

[1] T. Aubin, Problémes isopérimétriques et espaces de Sobolev. Journal of Differential Geometry, 11.4(1976), 573-598.

[2] T. Akahori, S. Ibrahim, H. Kikuchi and H. Nawa, Existence of a ground state and blow-up problem for a nonlinear Schrödinger equation with critical growth. Differential \& Integral Equations 25.3/4(2012), 383-402.

[3] T. Akahori, S. Ibrahim, H. Kikuchi and H. Nawa. Existence of a ground state and scattering for a nonlinear Schrödinger equation with critical growth. Selecta Mathematica New 19.2(2013), 545-609.

[4] T. Akahori, S. Ibrahim, H. Kikuchi and H. Nawa, Global dynamics above the ground state energy for the combined power-type nonlinear Schrödinger equations with energy-critical growth at low frequencies. arXiv: $1510.08034 \mathrm{v} 1$.

[5] J. Bourgain, Global wellposedness of defocusing critical nonlinear Schrödinger equation in the radial case. Journal of the American Mathematical Society. 12.1(1999), 145-171.

[6] T. Cazenave, Semilinear Schrödinger equations. Courant Lecture Notes in Mathematics, 10, NYU, CIMS, AMS 2003: 635-637.

[7] T. Cazenave and F. B. Weissler, The Cauchy problem for the nonlinear Schrödinger equation in $H^{1}$. Manuscripta Mathematica, 61.4(1988), 477-494.

[8] T. Cazenave and F. B. Weissler, The Cauchy problem for the critical nonlinear Schrödinger equation in $H^{s}$. Nonlinear Analysis, TMA, 14.10(1990), 807-836.

[9] M. Christ and M.I. Weistein, Dispersion of small amplitude solutions of the generalized Korteweg-de Vries equation. Journal of Functional Analysis, 100.1(1991), 87-109.

[10] J. Colliander, M. Keel, G. Staffilani, H. Takaoka and T. Tao, Global existence and scattering for rough solutions of a nonlinear Schrödinger equation on $\mathbb{R}^{3}$. Communications on Pure \& Applied Mathematics, 57.8(2004), 987-1014. 
[11] J. Colliander, M. Keel, G. Staffilani, H. Takaoka and T. Tao, Global well-posedness and scattering for the energy-critical nonlinear Schrödinger equation in $\mathbb{R}^{3}$. Annals of Mathematics, 167.3(2008), 767-865.

[12] B. Dodson, Global well-posedness and scattering for nonlinear Schrödinger equations with algebraic nonlinearity when $d=2,3, u_{0}$ radial. arXiv: 1405.0218

[13] B. Dodson, Global well-posedness and scattering for the focusing, energy-critical nonlinear Schrödinger problem in dimension $d=4$ for initial data below a ground state threshold. arXiv: 1409.1950

[14] B. Dodson, Global well-posedness and scattering for the defocusing, $L^{2}$-critical, nonlinear Schrödinger equation when $d=1$. American Journal of Mathematics 138.2 (2016), 531-569.

[15] J. Ginibre and G. Velo, Smoothing properties and retarded estimates for some dispersive evolution equations. Communications in Mathematical Physics, 144.1(1992), 163-188.

[16] S. Ibrahim, N. Masmoudi and K. Nakanishi, Scattering threshold for the focusing nonlinear Klein-Gordon equation. Analysis \& Partial Differential Equations, 4.3(2011), 405-460.

[17] M. Keel and T. Tao, Endpoint strichartz estimates. American Journal of Mathematics. 120.5(1998), 955-980.

[18] C. Kenig and F. Merle, Global well-posedness, scattering and blow-up for the energy-critical, focusing, non-linear Schrödinger equation in the radial case. Inventiones mathematicae, 166.3(2006), 645-675.

[19] R. Killip, T. Oh, O. Pocovnicu and M. Visan, Solitons and scattering for the cubic-quintic nonlinear Schrödinger equation on $\mathbb{R}^{3}$. Arch. Ration. Mech. Anal. 225.1(2017), 469-548.

[20] R. Killip and M. Visan, The focusing energy-critical nonlinear Schrödinger equation in dimensions five and higher. American Journal of Mathematics, 132.2(2010), 361-424.

[21] R. Killip and M. Visan, Global well-posedness and scattering for the defocusing quintic NLS in three dimensions. Analysis \& Partial Differential Equations, 5.4(2012), 855-885.

[22] S. Kerrani, On the defect of compactness for the Strichartz estimates of the Schrödinger Equations. Journal of Differential Equations, 175.2(2001), 353-392.

[23] C. Miao, G. Xu and L. Zhao, The dynamics of the 3D radial NLS with the combined terms. Communications in Mathematical Physics, 318.3(2013), 767-808.

[24] C. Miao, G. Xu and L. Zhao, The dynamics of the NLS with the combined terms in five and higher dimensions. Some Topics in Harmonic Analysis and Applications, 265-298, Advanced Lectures in Mathematics, ALM34, Higher Education Press, Beijing, and International Press, USA, 2015.

[25] K. Nakanishi, Energy scattering for nonlinear Klein-Gordon and Schrödinger equations in spatial dimensions 1 and 2. Journal of Functional Analysis, 169.1(1999), 201-225.

[26] K. Nakanishi, Remarks on the energy scattering for nonlinear Klein-Gordon and Schrödinger equations. Tohoku Mathematical Journal, Second Series 53.2 (2001), 285-303.

[27] E. Ryckman and M. Visan, Global well-posedness and scattering for the defocusing energy-critical nonlinear Schrödinger equation in $\mathbb{R}^{1+4}$. American Journal of Mathematics, 129.1(2007), 1-60.

[28] R. Strichartz, Restriction of Fourier transform to quadratic surfaces and decay of solutions of wave equations. Duke Mathematical Journal, 44.3(1977), 705-774.

[29] G. Talenti, Best constant in Sobolev inequality, Annali Di Matematica Pura Ed Applicata 110.1(1976), 353-372.

[30] T. Tao, M. Visan and X. Zhang, Global well-posedness and scattering for the defocusing mass-critical nonlinear Schrödinger equation for radial data in high dimensions. Duke Mathematical Journal, 140.1(2007), 165-202.

[31] T. Tao, M. Visan and X. Zhang, The nonlinear Schrödinger equation with combined powertype nonlinearities. Communications in Partial Differential Equations 32.8(2007), 1281-1343.

[32] M. Visan, The defocusing energy - critical nonlinear Schrödinger Equation in higher dimensions. Duke Mathematical Journal, 138.2(2007), 281-374.

[33] M. Visan, Global Well-posedness and Scattering for the Defocusing Cubic nonlinear Schrödinger equation in Four Dimensions. International Mathematics Research Notices 2012.5(2012), 1037-1067. 
Changxing Miao:

Institute of Applied Physics and Computational Mathematics,

P. O. Box 8009, Beijing, China, 100088,

E-mail address: miao_changxing@iapcm.ac.cn

Tengfei ZhaO:

The Graduate School of China Academy of Engineering Physics,

P. O. Box 2101, BeiJing, China, 100088,

E-mail address: zhao_tengfei@yeah.net

JiQIANG ZHENG

Université Nice Sophia-Antipolis,

06108 Nice Cedex 02, France

E-mail address: zhengjiqiang@gmail.com, zheng@unice.fr 